NONCOMMUTATIVE GEOMETRY

AND QUANTUM GROUPS

BANACH CENTER PUBLICATIONS, VOLUME 61

INSTITUTE OF MATHEMATICS

POLISH ACADEMY OF SCIENCES

WARSZAWA 2003

\title{
GENERALIZED SIGNATURE OPERATORS AND SPECTRAL TRIPLES FOR THE KRONECKER FOLIATION
}

\author{
R. MATTHES \\ Fachbereich 2, Technische Universität Clausthal \\ Leibnizstraße 4, 38678 Clausthal-Zellerfeld, Germany \\ E-mail: ptrm@pt.tu-clausthal.de \\ O. RICHTER and G. RUDOLPH \\ Institut für Theoretische Physik, Universität Leipzig \\ Augustusplatz 10/11, 04109 Leipzig, Germany \\ E-mail: Olaf.Richter@itp.uni-leipzig.de,rudolph@rz.uni-leipzig.de
}

\begin{abstract}
We consider two spectral triples related to the Kronecker foliation. The corresponding generalized Dirac operators are constructed from first and second order signature operators. Furthermore, we consider the differential calculi corresponding to these spectral triples. In one case, the calculus has a description in terms of generators and relations, in the other case it is an "almost free" calculus.
\end{abstract}

1. Introduction. The notion of a spectral triple is fundamental in noncommutative differential geometry, see [4]. It encodes the Riemannian and differential structure of a noncommutative space as well as its dimension. Aiming at applications in physics, spectral triples have been used to construct unified field theoretical models, e.g., the standard model (see [4], [5]), and models including gravitation ([3], [13], [14]). A main achievement in this connection was the identification of the Higgs field as a gauge field originating from a noncommutative differential calculus on a discrete space. From the mathematical point of view, only a few types of noncommutative spaces have been used in these examples: commutative algebras of smooth functions on a manifold [4], finite dimensional algebras (for a classification of spectral triples in this case see [15] and [20]) and products of both. Further examples of spectral triples have also been described explicitly for the irrational rotation algebra and higher dimensional noncommutative tori [4], [11].

In order to formulate gauge field theories on noncommutative spaces one usually needs explicit information about the differential calculus of the corresponding spectral triple.

2000 Mathematics Subject Classification: 16W25, 81V15.

The paper is in final form and no version of it will be published elsewhere. 
Such calculi have been analyzed in the above-mentioned cases ([4], [5], [12], [16]). In [17] it has been shown that the extra structure of a finitely generated projective module allows to introduce the graded algebra of differential-form-valued endomorphisms. This gives a natural mathematical language to build unified field theoretical models in the spirit of the Mainz-Marseille approach [7].

We remark that, using ideas from supersymmetric quantum theory, the notion of a spectral triple itself has been modified and enriched, see [8] and [9]. This leads to noncommutative generalizations of certain classical geometrical structures (Riemannian, symplectic, Hermitian, Kähler, ...). One hopes to apply these to superconformal field theories with noncommutative target spaces.

In the seminal paper [6] Connes and Moscovici have shown how to associate spectral triples to crossed product algebras related to foliations. Let $(M, \mathcal{F})$ be a regular foliation of a smooth manifold $M$ with Euclidean structures on both the corresponding distribution and the normal bundle. Then, there is an associated spectral triple for the crossed product algebra $C^{\infty}(M) \rtimes \Gamma$, where $\Gamma$ is a group of diffeomorphisms preserving these structures. The corresponding Dirac operator is hypoelliptic and closely related to the signature operator of the foliated manifold (a slight modification of the standard signature operator in differential geometry, see [10]).

In [18], we have given two examples of spectral triples related to the Kronecker foliation, partly following the method of [6]. Moreover, we gave a description of the corresponding differential calculi. The aim of the present paper is to give details of the proofs of the main propositions about the differential calculi. For the convenience of the reader, we start with a short review of the general notion of a spectral triple and of the construction of such triples related to foliations by the general method of [6]. We continue reviewing the construction of two spectral triples related to the Kronecker foliation given in [18]. This foliation is defined by an action of $\mathbb{R}$ on $\mathbb{T}^{2}$ which obviously preserves natural translation-invariant Euclidean structures on the distribution as well as on the normal bundle. We take $\Gamma=\mathbb{R}$ and arrive at the algebra $C^{\infty}\left(\mathbb{T}^{2}\right) \rtimes \mathbb{R}$, whose $C^{*}$-version is Morita equivalent to the irrational rotation algebra (noncommutative torus), see [22], [11]. The Dirac operator of the first spectral triple (which has dimension 2) is closely related to the ordinary signature operator on $\mathbb{T}^{2}$. The construction of the second triple (of dimension three) follows the strategy proposed in [6]. The corresponding signature operators, and henceforth also the Dirac operators, can be diagonalized explicitly in both cases. Then we pass to the differential calculi associated to the above spectral triples. For the triple related to the first order signature operator the differential calculus can be completely characterized in terms of generators and relations. The restriction to $\Gamma=\mathbf{1}$ leads to the de Rham calculus on $\mathbb{T}^{2}$. The analysis of the differential calculus for the second triple turns out to be much more involved. We show that in this case the restriction to $\Gamma=\mathbf{1}$ gives just the universal calculus on $C^{\infty}\left(\mathbb{T}^{2}\right)$. For the case $\Gamma=\mathbb{R}$, we conjecture that an "almost free" calculus is obtained.

2. The spectral triple related to a foliation. For the convenience of the reader, we recall here the definition of a spectral triple and the differential calculus related to such a triple ([4], [11]): 
Definition 1. A spectral triple $(A, \mathcal{H}, D)$ consists of a $*$-algebra $A$, a Hilbert space $\mathcal{H}$ and an unbounded selfadjoint operator $D$ on $\mathcal{H}$, such that

(i) $A$ acts on $\mathcal{H}$ by a $*$-representation $\pi: A \rightarrow B(\mathcal{H})(B(\mathcal{H})$ - the algebra of bounded operators on $\mathcal{H}$ ),

(ii) the commutators $[D, \pi(a)], a \in A$, are bounded and

(iii) the operator $D$ has discrete spectrum with finite multiplicity.

A spectral triple has dimension $n$ if the eigenvalues (counted with multiplicity) $\mu_{k}$ of $|D|$ fulfil $\lim _{k \rightarrow \infty} \mu_{k} / k^{1 / n}=C \neq 0$.

We will not refer to gradings or real structures usually included in the definition of a spectral triple, nor to more general notions of dimension.

The representation $\pi$ of $A$ in $B(\mathcal{H})$ can be extended to a representation $\pi^{*}: \Omega(A) \rightarrow$ $B(\mathcal{H})$ of the universal differential calculus $\Omega(A)$ by

$$
\pi^{n}\left(\sum_{k} a_{0}^{k} d a_{1}^{k} \cdots d a_{n}^{k}\right)=\sum_{k} \pi\left(a_{0}^{k}\right)\left[D, \pi\left(a_{k}^{1}\right)\right] \cdots\left[D, \pi\left(a_{n}^{k}\right)\right] .
$$

If $J_{0}:=\oplus_{n} \operatorname{ker} \pi^{n}$, then $J:=J_{0}+d J_{0}$ is a differential ideal, and one arrives at the differential calculus $\Omega_{D}(A)$,

$$
\Omega_{D}^{n}(A):=\Omega^{n}(A) / J .
$$

Note that, if $\pi$ is faithful, there are isomorphisms

$$
\Omega_{D}^{1}(A) \simeq \pi^{1}\left(\Omega^{1}(A)\right)
$$

and

$$
\Omega_{D}^{2}(A) \simeq \pi^{2}\left(\Omega^{2}(A)\right) / \pi^{2}\left(d J_{0}^{1}\right) .
$$

Now we review briefly the procedure given in [6], which relates a spectral triple to a regular foliation of a smooth manifold. Let $M$ be a compact manifold with a foliation given by an integrable distribution $V \subset T M$. The normal bundle of the foliation is $N:=$ $T M / V$, with canonical projection $\rho: T M \rightarrow N$. Assume further that both $V$ and $N$ are equipped with Euclidean fibre metrics and with an orientation (i.e., distinguished nowhere vanishing sections $\omega_{V}, \omega_{N}$ of the exterior bundles $\left.\bigwedge^{v} V, \bigwedge^{n} N(v=\operatorname{dim} V, n=\operatorname{dim} N)\right)$. The orientations $\omega_{V}$ and $\omega_{N}$ also define a nonvanishing section of $\bigwedge^{v} V^{*} \otimes \bigwedge^{n} N^{*} \simeq$ $\bigwedge^{v+n} T^{*} M$, i.e. a volume form on $M$. Let us consider the bundle

$$
E=\bigwedge V_{\mathbb{C}}^{*} \otimes \bigwedge N_{\mathbb{C}}^{*}
$$

(complexification). The metrics on $V$ and $N$ give rise to Hermitian metrics on $\bigwedge V_{\mathbb{C}}^{*}$ and $\bigwedge N_{\mathbb{C}}^{*}$ and thus also on $E$. The orientations $\omega_{V}$ and $\omega_{N}$ can be mapped by means of the metrics to sections $\gamma_{V}$ of $\bigwedge^{v} V_{\mathbb{C}}^{*}$ and $\gamma_{N}$ of $\bigwedge^{n} N_{\mathbb{C}}^{*}$ which can be used, together with the metrics, to define an analogue of the Hodge star on the exterior bundles $\bigwedge V_{\mathbb{C}}^{*}$ and $\bigwedge N_{\mathbb{C}}^{*}$. We choose a variant of the $*$-operation such that $*_{V_{\mathbb{C}}}^{2}=1$ and $*_{N_{\mathbb{C}}}^{2}=1$, i.e. $* V_{\mathbb{C}}$ and $*_{N_{\mathbb{C}}}$ can be considered as $\mathbb{Z}_{2}$-grading operators (cf. [1]).

Thus, the space of sections of $E$ has a natural inner product, and we denote by $\mathcal{H}=L^{2}(M, E)$ the Hilbert space of square integrable sections of this bundle. From now on, we always consider complexified vector bundles, but omit the subscript $\mathbb{C}$. 
In order to construct a generalized Dirac operator, a longitudinal differential $d_{L}$ and a transversal differential operator $d_{H}$ have to be defined. The differential $d_{L}$ is defined canonically by means of the Bott connection $([2])$ given as the partial covariant derivative $\nabla: \Gamma(V) \times \Gamma(N) \rightarrow \Gamma(N)$ defined by

$$
\nabla_{X} Y=\rho([X, \tilde{Y}])
$$

for $X \in \Gamma(V), Y \in \Gamma(N)$ and $\tilde{Y} \in \Gamma(T M)$ such that $\rho(\tilde{Y})=Y$. By a standard procedure (using the Leibniz rule and duality) $\nabla$ is extended to a differential $d_{L}: \Gamma(E) \rightarrow \Gamma(E)$ defined by linear mappings $\Gamma\left(\bigwedge^{k} V^{*} \otimes \bigwedge^{l} N^{*}\right) \rightarrow \Gamma\left(\bigwedge^{k+1} V^{*} \otimes \bigwedge^{l} N^{*}\right)$,

$$
\begin{aligned}
d_{L} \alpha\left(X_{0}, \ldots, X_{k}\right) & =\sum_{i=0, \ldots, k}(-1)^{i} \nabla_{X_{i}}\left(\alpha\left(X_{0}, \ldots, \hat{X}_{i}, \ldots, X_{k}\right)\right) \\
& +\sum_{i<j}(-1)^{i+j} \alpha\left(\left[X_{i}, X_{j}\right], X_{0}, \ldots, \hat{X}_{i}, \ldots, \hat{X}_{j}, \ldots, X_{k}\right),
\end{aligned}
$$

$X_{i} \in \Gamma(V)$. Since the Bott connection is flat, we have $d_{L}^{2}=0$.

In order to define a transversal differential operator one has to choose a subbundle $H \subset T M$ complementary to $V$. This defines a bundle isomorphism $j_{H}: \bigwedge V^{*} \otimes \wedge N^{*} \rightarrow$ $\bigwedge T^{*} M$ in the following way: Let us denote by $\operatorname{pr}_{V}^{*}$ and $\operatorname{pr}_{H}^{*}$ the projections corresponding to the decomposition $T M^{*}=V^{*} \oplus H^{*}$, by $\rho_{H}: H \rightarrow N$ the restriction of $\rho$ to $H$ and by $\rho_{H}^{*}$ its transposed map. Then $j_{H}$ is defined as the following composition:

$$
\bigwedge V^{*} \otimes \bigwedge N^{*} \stackrel{\operatorname{id} \otimes \wedge \rho_{H}^{*}}{\longrightarrow} \bigwedge V^{*} \otimes \bigwedge H^{*} \stackrel{\wedge \operatorname{pr}_{V}^{*} \otimes \wedge \operatorname{pr}_{H}^{*}}{\longrightarrow} \bigwedge T^{*} M \otimes \bigwedge T^{*} M \stackrel{\otimes \rightarrow \wedge}{\longrightarrow} \bigwedge T^{*} M
$$

where $\otimes \rightarrow \wedge$ denotes the replacement of the tensor product by the wedge product. Now, the transversal operator $d_{H}$ is obtained from the exterior differential $d$ by transporting with $j_{H}$ and projecting to a certain homogeneous component: $\bigwedge V^{*} \otimes \wedge N^{*}$ has an obvious bigrading, and denoting by $\pi^{(r, s)}$ the projector to the homogeneous component of bidegree $(r, s)$, one defines

$$
d_{H} \alpha=\pi^{(r, s+1)}\left(j_{H}^{-1} \circ d \circ j_{H}(\alpha)\right)
$$

for $\alpha \in \Gamma\left(\bigwedge^{r} V^{*} \otimes \bigwedge^{s} N^{*}\right)$. The operator $d_{H}$ is a graded derivation of the $\mathbb{Z}_{2}$-graded algebra $\Gamma\left(\bigwedge V^{*} \otimes \bigwedge N^{*}\right)$.

In a foliation chart, $d_{L}$ and $d_{H}$ look as follows. Let $\left(x^{i}, y^{k}\right), i=1, \ldots, v, k=1, \ldots, n$ be local coordinates of $M$ such that $x^{i}$ are coordinates on the leaf for fixed $y^{k}$ (foliation chart). The corresponding coordinate vector fields $\left(\partial / \partial x^{i}, \partial / \partial y^{k}\right)$ form a local frame of $T M$ and $\left(\partial / \partial x^{i}\right)$ a frame of $V$. The corresponding dual frame of $T^{*} M$ consists of the differentials $\left(d x^{i}, d y^{k}\right)$. We define $\theta^{i} \in \Gamma\left(V^{*}\right)$ by $\theta^{i}\left(\partial / \partial x^{j}\right)=\delta_{j}^{i}(i, j=1, \ldots, v)$. It is immediate from the definition of $N$ that the elements $n_{k}:=\partial / \partial y^{k}+V(k=1, \ldots, n)$ form a local frame of $N$. The elements of the corresponding dual frame of $N^{*}$ are denoted by $n^{k}$. Finally, we choose a local frame $h_{k}$ of the transversal space $H$. This frame is fixed by assuming $\rho_{H}\left(h_{k}\right)=n_{k}$. This leads to

$$
h_{k}=h_{k}^{i} \frac{\partial}{\partial x^{i}}+\frac{\partial}{\partial y^{k}},
$$

with coefficient functions $h_{k}^{i}$ characterizing $H$. Then, the elements $\theta^{i_{1}} \wedge \cdots \wedge \theta^{i_{r}} \otimes n^{j_{1}} \wedge$ $\cdots \wedge n^{j_{s}}$ form a local frame of $E$, and one can show that $d_{L}$ and $d_{H}$ are given by the 
following local formulae:

$$
\begin{gathered}
d_{L}\left(\alpha_{i_{1} \ldots i_{r} j_{1} \ldots j_{s}} \theta^{i_{1}} \wedge \cdots \wedge \theta^{i_{r}} \otimes n^{j_{1}} \wedge \cdots \wedge n^{j_{s}}\right)=\frac{\partial \alpha_{i_{1} \ldots i_{r} j_{1} \ldots j_{s}}}{\partial x^{i}} \theta^{i} \wedge \theta^{i_{1}} \wedge \cdots \theta^{i_{r}} \otimes n^{j_{1}} \wedge \cdots \wedge n^{j_{s}}, \\
\quad d_{H}\left(\alpha_{i_{1} \ldots i_{r} j_{1} \ldots j_{s}} \theta^{i_{1}} \wedge \cdots \wedge \theta^{i_{r}} \otimes n^{j_{1}} \wedge \cdots \wedge n^{j_{s}}\right)= \\
(-1)^{r} h_{k}\left(\alpha_{i_{1} \ldots i_{r} j_{1} \ldots j_{s}}\right) \theta^{i_{1}} \wedge \cdots \wedge \theta^{i_{r}} \otimes n^{k} \wedge n^{j_{1}} \wedge \cdots \wedge n^{j_{s}}+ \\
\alpha_{i_{1} \ldots i_{r} j_{1} \ldots j_{s}} \sum_{t=1}^{r} \frac{\partial h_{k}^{i_{t}}}{\partial x^{l}} \theta^{i_{1}} \wedge \cdots \wedge \theta^{l} \wedge \cdots \wedge \theta^{i_{r}} \otimes n^{k} \wedge n^{j_{1}} \wedge \cdots \wedge n^{j_{s}}
\end{gathered}
$$

(where $\theta^{l}$ at position $t$ replaces $\theta^{i_{t}}$ ). The longitudinal differential $d_{L}$ acts as a differential in leaf direction, whereas $d_{H}$ is a sum of a principal part, which differentiates in transversal direction, and a zero order part. As examples, let us give formulae for $d_{H}$ acting on functions, (1,0)-, (0,1)- and (1,1)-forms:

$$
\begin{aligned}
d_{H} f & =h_{k}(f) n^{k} \\
d_{H}\left(\alpha_{i} \theta^{i}\right) & =-\left(h_{k}\left(\alpha_{j}\right)+\alpha_{i} \frac{\partial h_{k}^{i}}{\partial x^{j}}\right) \theta^{j} \otimes n^{k}, \\
d_{H}\left(\alpha_{k} n^{k}\right) & =h_{l}\left(\alpha_{k}\right) n^{l} \wedge n^{k}, \\
d_{H}\left(\alpha_{i k} \theta^{i} \wedge n^{k}\right) & =-\left(h_{l}\left(\alpha_{j k}\right)+\alpha_{i k} \frac{\partial h_{l}^{i}}{\partial x^{j}}\right) \theta^{j} \otimes n^{l} \wedge n^{k},
\end{aligned}
$$

$\left(d_{H}\left(n^{k}\right)=0\right)$. For the adjoint operators $d_{L}^{*}$ and $d_{H}^{*}($ in $\mathcal{H})$ it is difficult to write down explicit formulae. One can show

$$
d_{L}^{*} \alpha=-*_{V} d_{L} *_{V}+\text { term of order zero, }
$$

where $*_{V}$ is the (partial) Hodge operator related to the Euclidean metric and the orientation of $V$. Since $d_{H}^{*}$ lowers the $N^{*}$-degree one has for $\alpha \in \Gamma\left(\bigwedge^{r} V^{*}\right) \equiv \Gamma\left(\bigwedge^{r} V^{*} \otimes \bigwedge^{0} N^{*}\right)$

$$
d_{H}^{*} \alpha=0 .
$$

Explicit formulae for $d_{H}^{*}$ become rather complicated as, e.g., the case of $(0,1)$-forms shows:

$$
d_{H}^{*}\left(\alpha_{i} \theta^{i}\right)=-g_{N}^{k l}\left(h_{k}\left(\alpha_{l}\right)-\alpha_{m} \Gamma_{N k l}^{m}+\alpha_{l}\left(\frac{\partial h_{k}^{i}}{\partial x^{i}}+\frac{1}{2} g_{V}^{i j} h_{k}\left(g_{V i j}\right)\right),\right.
$$

where $g_{N}^{k l}=g_{N}\left(n^{k}, n^{l}\right), g_{V i j}=g_{V}\left(\partial / \partial x^{i}, \partial / \partial x^{j}\right), g_{V}^{i j}=g_{V}\left(\theta^{i}, \theta^{j}\right)$ are the local components of the fibre metrics (and their duals), and $\Gamma_{N}^{m}$ are the "Christoffel symbols" corresponding to $g_{N} k l$.

In [6], using $d_{H}$ and $d_{L}$, two differential operators are introduced by

$$
Q_{L}=d_{L} d_{L}^{*}-d_{L}^{*} d_{L}, \quad Q_{H}=d_{H}+d_{H}^{*},
$$

and the mixed signature operator $Q$ for $M$, acting on a form with $N$-degree $\partial_{N}$, is defined by

$$
Q=Q_{L}(-1)^{\partial_{N}}+Q_{H}
$$

As noted in [6], $Q$ is selfadjoint. Finally, a generalized Dirac operator $D$ is defined as the unique selfadjoint operator such that

$$
D|D|=Q
$$


If zero is not an element of the spectrum of $Q$, it is given as

$$
D=Q|Q|^{-1 / 2}=Q\left(Q^{2}\right)^{-1 / 4}
$$

as shows a straightforward argument using the spectral decomposition of $Q$.

One motivation for choosing a second order longitudinal part is the following: the index of the signature operator should not depend on the choice of the transversal subbundle $H$. Usually, the index of a pseudodifferential operator only depends on its principal symbol. However, as follows from the local formulae (2.3) and (2.4), its principal part explicitly depends on $H$, the dependence being in the coefficients of the partial derivatives with respect to leaf coordinates. It turns out that one can get rid of this dependence by introducing a modified notion of pseudodifferential operators $\left(\psi D O^{\prime}\right)$ which assigns a degree 2 to transversal coordinates and a degree 1 to longitudinal ones. To have a contribution also from $Q_{L}$, one has to pass to a second order operator. In [6] a homotopy argument was given to show that this does not affect the longitudinal signature class.

Let $\Gamma$ be any group of diffeomorphisms of $M$ which preserves the distribution $V$ and the Euclidean metrics on both $V$ and $N$. Then $\psi \in \Gamma$ acts via the pull back as unitary operator $U_{\psi}^{*}$ on $\mathcal{H}$, whereas functions from $C^{\infty}(M)$ act there as multiplication operators. The crossed product algebra $\mathcal{A}:=C^{\infty}(M) \rtimes \Gamma$ can be defined as the $*$-subalgebra of $B(\mathcal{H})$ generated by these two types of operators. Due to $U_{\psi}^{*} f=(f \circ \psi) U_{\psi}^{*}$ every element of $\mathcal{A}$ is a finite sum of elements $f U_{\psi}^{*}$. Then we have, see [6]:

TheOREM 1. $(\mathcal{A}, \mathcal{H}, D)$ is a spectral triple of dimension $v+2 n$.

One of our main aims is to describe explicitly this spectral triple for the Kronecker foliation of the two-torus.

\section{Spectral triples for the Kronecker foliation}

3.1. Crossed product algebra and Hilbert space. We consider the two-torus as the quotient $\mathbb{T}^{2}=\mathbb{R}^{2} / 2 \pi \mathbb{Z}^{2}$. Thus, we have natural local coordinates $0<\vartheta_{1}, \vartheta_{2}<2 \pi$. Consider the $\mathbb{R}$-manifold $\left(\mathbb{T}^{2}, \mathbb{R}, \psi\right)$, with group action

$$
\psi: \mathbb{T}^{2} \times \mathbb{R} \rightarrow \mathbb{T}^{2},
$$

given by

$$
\psi\left(\left(\vartheta_{1}, \vartheta_{2}\right), t\right)=\left(\vartheta_{1}+a t, \vartheta_{2}+b t\right)
$$

with $a, b \in \mathbb{R}$ such that $a>0, a^{2}+b^{2}=1$ and $\theta=b / a$ being irrational. The foliation of $\mathbb{T}^{2}$ by the orbits of $\psi$ is called the Kronecker foliation. It is well known, see [19], that each leaf of this foliation is diffeomorphic to $\mathbb{R}$ and lies dense in $\mathbb{T}^{2}$.

The coordinate transformation

$$
\begin{aligned}
& x=a \vartheta_{1}+b \vartheta_{2}, \\
& y=b \vartheta_{1}-a \vartheta_{2}
\end{aligned}
$$

is orthogonal and leads to coordinates $(x, y)$ of a foliation chart. In these coordinates, $\mathbb{R}$ acts as follows:

$$
\psi((x, y), t)=(x+t, y)
$$


To be more precise, this is the lifted action of $\mathbb{R}$ on $\mathbb{R}^{2}$, applied to global coordinates $(x, y)$ obtained from global coordinates $\left(\vartheta_{1}, \vartheta_{2}\right)$ by the orthogonal transformation.

The associated crossed product algebra

$$
\mathcal{O}=\mathcal{O}\left(\mathbb{T}^{2}\right) \rtimes \mathbb{R}
$$

is the *-algebra generated by the unitary operators $U_{1}, U_{2}$ and $V_{t}$ acting in the Hilbert space $L^{2}\left(\mathbb{T}^{2}\right)$ given by

$$
\begin{aligned}
& \left(U_{1} \xi\right)\left(\vartheta_{1}, \vartheta_{2}\right)=\mathrm{e}^{\mathrm{i} \vartheta_{1}} \cdot \xi\left(\vartheta_{1}, \vartheta_{2}\right), \\
& \left(U_{2} \xi\right)\left(\vartheta_{1}, \vartheta_{2}\right)=\mathrm{e}^{\mathrm{i} \vartheta_{2}} \cdot \xi\left(\vartheta_{1}, \vartheta_{2}\right), \\
& \left(V_{t} \xi\right)\left(\vartheta_{1}, \vartheta_{2}\right)=\xi\left(\vartheta_{1}+a t, \vartheta_{2}+b t\right),
\end{aligned}
$$

$\forall \xi \in L^{2}\left(T^{2}\right)$. Let $e_{k l}=\mathrm{e}^{\mathrm{i}\left(k \vartheta_{1}+l \vartheta_{2}\right)}(k, l \in \mathbb{Z})$ be the basis of trigonometric polynomials of $L^{2}\left(\mathbb{T}^{2}\right)$. It follows from $(3.1)$ that

$$
\begin{aligned}
U_{1} e_{k l} & =e_{k+1, l}, \\
U_{2} e_{k l} & =e_{k, l+1}, \\
V_{t} e_{k l} & =\mathrm{e}^{\mathrm{i}(a k+b l) t} e_{k l} .
\end{aligned}
$$

It is now immediate to show

Proposition 1. The unitary operators $U_{1}, U_{2}, V_{t}$ satisfy

$$
\begin{aligned}
U_{1} U_{2} & =U_{2} U_{1}, \\
V_{t} U_{1} & =\mathrm{e}^{\mathrm{i} a t} U_{1} V_{t}, \\
V_{t} U_{2} & =\mathrm{e}^{\mathrm{i} b t} U_{2} V_{t}, \\
V_{t} V_{s} & =V_{t+s}, t, s \in \mathbb{R} .
\end{aligned}
$$

REMARK 1. For rational $\frac{a}{b}=\frac{m}{n}, m, n$ relatively prime, the operators $U_{1}, U_{2}, V_{t}$ are also well-defined. In this case, there is an additional relation

$$
V_{2 \pi \sqrt{m^{2}+n^{2}}}=V_{0}=1 ;
$$

$2 \pi \sqrt{m^{2}+n^{2}}$ is the smallest value of $t$ such that $V_{t}=V_{0}=1$; any other such $t$ is an integer multiple of this value.

Proposition 2. The *-algebra $\mathcal{O}\left(\mathbb{T}^{2}\right) \rtimes \mathbb{R}$ is isomorphic to $\mathbb{C}\left\langle u_{1}, u_{2}, v_{t}\right\rangle / J$, where $\mathbb{C}\left\langle u_{1}, u_{2}, v_{t}\right\rangle$ is the free associative unital $*$-algebra generated by $u_{1}, u_{2}$ and $v_{t}, t \in \mathbb{R}$, and $J$ is the *-ideal generated by (3.3)-(3.6) and unitarity conditions for the generators.

Proof. See [18].

In analogy with the definition given before Theorem 1 , putting $M=\mathbb{T}^{2}$ and $\Gamma=\mathbb{R}$, we define the crossed product

$$
\mathcal{A}:=C^{\infty}\left(\mathbb{T}^{2}\right) \rtimes \mathbb{R}
$$

as a $*$-subalgebra of $B\left(L^{2}\left(\mathbb{T}^{2}\right)\right)$. As noted in [18, Remark 2], $\mathcal{A}$ can be considered as a topological completion of the $*$-algebra $\mathcal{O}$ in a natural Fréchet topology.

In our concrete case, the Hilbert space of the spectral triple of Theorem 1 can be described as follows: Both $V$ and $N$ are one-dimensional, with local frames consisting each 
of one vector $\partial / \partial x$ and $\underline{n}=\partial / \partial y+V$ respectively. Let $\tau$ and $\nu$ denote the corresponding elements of the dual frames. Then $E=\bigwedge V^{*} \otimes \wedge N^{*}$ is the direct sum of four line bundles of elements of degrees $(0,0),(1,0),(0,1),(1,1)$, with local frames $\mathbf{1}, \tau, \nu, \tau \otimes \nu$, respectively. The natural choice of translation invariant (under the natural action of $\mathbb{R}^{2}$ on $\mathbb{T}^{2}$ ) Euclidean fibre metrics makes these frame elements mutually orthogonal unit vectors in $L^{2}\left(\mathbb{T}^{2}, E\right)$. Note that all fibre metrics that are invariant under the $\mathbb{R}$-action defining the foliation are also translation invariant under $\mathbb{R}^{2}$ (see $[18$, Lemma 1]). We may identify

$$
L^{2}\left(\mathbb{T}^{2}, E\right)=L^{2}\left(T^{2}\right) \oplus L^{2}\left(T^{2}\right) \oplus L^{2}\left(T^{2}\right) \oplus L^{2}\left(T^{2}\right),
$$

with $e_{k l} 1 \rightarrow\left(e_{k l}, 0,0,0\right), \ldots, e_{k l} \tau \otimes \nu \rightarrow\left(0,0,0, e_{k l}\right)$.

Since the generators act, according to $(3.1)$, componentwise in $L^{2}\left(\mathbb{T}^{2}, E\right)$ the crossed product algebra of Theorem 1 coincides with (3.7).

We choose the transversal subspace $H$ as simple as possible, i.e. we put $h_{k}^{i}=0$. Equivalently, $H$ is generated by the coordinate vector field $\partial / \partial y$. Then the general formulae of the foregoing section (and some easy computations for the adjoints) lead to the following expressions:

\begin{tabular}{c|c|c|c|c} 
& $f$ & $f \tau$ & $f \nu$ & $f \tau \otimes \nu$ \\
\hline$d_{L}$ & $\frac{\partial f}{\partial x}$ & 0 & $\frac{\partial f}{\partial x} \tau \otimes \nu$ & 0 \\
$d_{H}$ & $\frac{\partial f}{\partial y} \nu$ & $-\frac{\partial f}{\partial y} \tau \otimes \nu$ & 0 & 0 \\
$d_{L}^{*}$ & 0 & $-\frac{\partial f}{\partial x}$ & 0 & $-\frac{\partial f}{\partial x} \nu$ \\
$d_{H}^{*}$ & 0 & 0 & $-\frac{\partial f}{\partial y}$ & $\frac{\partial f}{\partial y} \tau$
\end{tabular}

with $f \in C^{\infty}\left(\mathbb{T}^{2}\right)$. To prove, e.g.,

$$
d_{H}^{*}(f \tau \otimes \nu)=\frac{\partial f}{\partial y} \tau,
$$

we denote by $(\cdot \mid \cdot)$ the scalar product in $L^{2}\left(\mathbb{T}^{2}, E\right)$ and observe that

$$
\begin{aligned}
\left(g \tau \mid d_{H}^{*}(f \tau \otimes \nu)\right) & \equiv\left(d_{H}(g \tau) \mid f \tau \otimes \nu\right)=\left(-\frac{\partial g}{\partial y} \tau \otimes \nu \mid f \tau \otimes \nu\right) \\
& =-\int \frac{\partial g(x, y)}{\partial y} f(x, y) d x d y=\int g(x, y) \frac{\partial f(x, y)}{\partial y} d x d y=\left(g \tau \mid \frac{\partial f}{\partial y} \tau\right) .
\end{aligned}
$$

Note that all the above operators can also be written as matrix differential operators.

3.2. The first order signature operator as Dirac operator. We will first show that $\left(C^{\infty}\left(\mathbb{T}^{2}\right) \rtimes \mathbb{R}, L^{2}\left(\mathbb{T}^{2}, E\right), \tilde{Q}\right)$, with $\tilde{Q}$ being the linear signature operator

$$
\tilde{Q}=d_{L}+d_{L}^{*}+d_{H}+d_{H}^{*},
$$

is a spectral triple of dimension 2. Using the foliation chart $(x, y)$ and the local frame $\{\mathbf{1}, \tau, \nu, \tau \otimes \nu\}$, this operator can be written as 


$$
\tilde{Q}=\left(\begin{array}{cccc}
0 & -\frac{\partial}{\partial x} & -\frac{\partial}{\partial y} & 0 \\
\frac{\partial}{\partial x} & 0 & 0 & \frac{\partial}{\partial y} \\
\frac{\partial}{\partial y} & 0 & 0 & -\frac{\partial}{\partial x} \\
0 & -\frac{\partial}{\partial y} & \frac{\partial}{\partial x} & 0
\end{array}\right)
$$

The eigenvalue problem for $\tilde{Q}$ is most easily solved by considering its square

$$
\tilde{Q}^{2}=\left(\begin{array}{cccc}
-\frac{\partial^{2}}{\partial x^{2}}-\frac{\partial^{2}}{\partial y^{2}} & 0 & 0 & 0 \\
0 & -\frac{\partial^{2}}{\partial x^{2}}-\frac{\partial^{2}}{\partial y^{2}} & 0 & 0 \\
0 & 0 & -\frac{\partial^{2}}{\partial x^{2}}-\frac{\partial^{2}}{\partial y^{2}} & 0 \\
0 & 0 & 0 & -\frac{\partial^{2}}{\partial x^{2}}-\frac{\partial^{2}}{\partial y^{2}}
\end{array}\right) .
$$

In coordinates $\left(\vartheta_{1}, \vartheta_{2}\right)$ one is immediately led to the eigenvalue equations

$$
\left(-\left(a \frac{\partial}{\partial \vartheta_{1}}+b \frac{\partial}{\partial \vartheta_{2}}\right)^{2}-\left(b \frac{\partial}{\partial \vartheta_{1}}-a \frac{\partial}{\partial \vartheta_{2}}\right)^{2}\right) f_{j}=\lambda^{2} f_{j}
$$

for the four components of an eigenvector $f=f_{1}+f_{2} \tau+f_{3} \nu+f_{4} \tau \otimes \nu$ of $\tilde{Q}^{2}$. It is now straightforward to see that

$$
e_{k l}^{1}=\left(\begin{array}{c}
e_{k l} \\
0 \\
0 \\
0
\end{array}\right), \quad e_{k l}^{2}=\left(\begin{array}{c}
0 \\
e_{k l} \\
0 \\
0
\end{array}\right), \quad e_{k l}^{3}=\left(\begin{array}{c}
0 \\
0 \\
e_{k l} \\
0
\end{array}\right), \quad e_{k l}^{4}=\left(\begin{array}{c}
0 \\
0 \\
0 \\
e_{k l}
\end{array}\right)
$$

are eigenvectors of $\tilde{Q}^{2}$ with the eigenvalue

$$
\lambda_{k l}^{2}=(a k+b l)^{2}+(a l-b k)^{2}=k^{2}+l^{2} .
$$

The operator $\tilde{Q}$ itself acts in this basis of $L^{2}\left(\mathbb{T}^{2}, E\right)$ as follows:

$$
\begin{aligned}
& \tilde{Q}\left(e_{k l}^{1}\right)=(a k+b l) e_{k l}^{2}+(a l-b k) e_{k l}^{3}, \\
& \tilde{Q}\left(e_{k l}^{2}\right)=(-a k-b l) e_{k l}^{1}+(-a l+b k) e_{k l}^{4}, \\
& \tilde{Q}\left(e_{k l}^{3}\right)=(-a l+b k) e_{k l}^{1}+(a k+b l) e_{k l}^{4}, \\
& \tilde{Q}\left(e_{k l}^{4}\right)=(a l-b k) e_{k l}^{2}-(a k+b l) e_{k l}^{3} .
\end{aligned}
$$

Thus, we have

$$
\lambda_{k l}^{ \pm}= \pm \sqrt{(a k+b l)^{2}+(a l-b k)^{2}}= \pm \sqrt{k^{2}+l^{2}}
$$

and

$$
\begin{aligned}
& e_{k l}^{+1}=-i \frac{b k-a l}{\lambda_{k l}^{+}} e_{k l}^{1}+e_{k l}^{3}+i \frac{a k+b l}{\lambda_{k l}^{+}} e_{k l}^{4}, \\
& e_{k l}^{+2}=-i \frac{a k+b l}{\lambda_{k l}^{+}} e_{k l}^{1}+e_{k l}^{2}+i \frac{a l-b k}{\lambda_{k l}^{+}} e_{k l}^{4}, \\
& e_{k l}^{-1}=e_{k l}^{1}-i \frac{a k+b l}{\lambda_{k l}^{+}} e_{k l}^{2}+i \frac{a l-b k}{\lambda_{k l}^{+}} e_{k l}^{3}, \\
& e_{k l}^{-2}=i \frac{a l-b k}{\lambda_{k l}^{+}} e_{k l}^{2}+i \frac{a k+b l}{\lambda_{k l}^{+}} e_{k l}^{3}+e_{k l}^{4}
\end{aligned}
$$

form a complete set of eigenvectors of $\tilde{Q}$. 
Proposition 3. $\left(C^{\infty}\left(\mathbb{T}^{2}\right) \rtimes \mathbb{R}, L^{2}\left(\mathbb{T}^{2}, E\right), \tilde{Q}\right)$ is a spectral triple of dimension 2.

Proof. See [18].

Note that the commutators of $\tilde{Q}$ with the generators $U_{1}$ and $U_{2}$ are explicitly given by

$$
\begin{aligned}
& {\left[\tilde{Q}, U_{1}\right] e_{k l}^{1}=a e_{k+1, l}^{2}-b e_{k+1, l}^{3},} \\
& {\left[\tilde{Q}, U_{1}\right] e_{k l}^{2}=-a e_{k+1, l}^{1}+b e_{k+1, l}^{4},} \\
& {\left[\tilde{Q}, U_{1}\right] e_{k l}^{3}=b e_{k+1, l}^{1}+a e_{k+1, l}^{4},} \\
& {\left[\tilde{Q}, U_{1}\right] e_{k l}^{4}=-b e_{k+1, l}^{2}-a e_{k+1, l}^{3}}
\end{aligned}
$$

and

$$
\begin{aligned}
& {\left[\tilde{Q}, U_{2}\right] e_{k l}^{1}=b e_{k, l+1}^{2}+a e_{k, l+1}^{3},} \\
& {\left[\tilde{Q}, U_{2}\right] e_{k l}^{2}=-b e_{k, l+1}^{1}-a e_{k, l+1}^{4},} \\
& {\left[\tilde{Q}, U_{2}\right] e_{k l}^{3}=-a e_{k, l+1}^{1}+b e_{k, l+1}^{4},} \\
& {\left[\tilde{Q}, U_{2}\right] e_{k l}^{4}=a e_{k, l+1}^{2}-b e_{k, l+1}^{3} .}
\end{aligned}
$$

In order to describe the differential algebra $\Omega_{\tilde{Q}}\left(\mathcal{O}\left(\mathbb{T}^{2}\right) \rtimes \mathbb{R}\right)$, we denote, as in formulae (2.1) and (2.2), by $\pi^{1}$ and $\pi^{2}$ the extensions of $\pi$ to universal one and two forms. Since $\pi$ is faithful by Proposition $2, \Omega_{\tilde{Q}}^{1}\left(\mathcal{O}\left(\mathbb{T}^{2}\right) \rtimes \mathbb{R}\right)$ is isomorphic to $\pi^{1}\left(\Omega^{1}\left(\mathcal{O}\left(\mathbb{T}^{2}\right) \rtimes \mathbb{R}\right)\right)$, with $d u_{j} \mapsto\left[\tilde{Q}, U_{j}\right], d v_{t} \mapsto\left[\tilde{Q}, V_{t}\right]$, and $\Omega_{\tilde{Q}}^{2}\left(\mathcal{O}\left(\mathbb{T}^{2}\right) \rtimes \mathbb{R}\right)=\Omega^{2}\left(\mathcal{O}\left(\mathbb{T}^{2}\right) \rtimes \mathbb{R}\right) /\left(\operatorname{ker} \pi^{2}+d\left(\operatorname{ker} \pi^{1}\right)\right) \simeq$ $\pi^{2}\left(\Omega^{2}\left(\mathcal{O}\left(\mathbb{T}^{2}\right) \rtimes \mathbb{R}\right)\right) / \pi^{2}\left(d\left(\operatorname{ker} \pi^{1}\right)\right)$.

Let us first note that, under the identification $L^{2}\left(\mathbb{T}^{2}, E\right) \simeq \mathbb{C}^{4} \otimes L^{2}\left(\mathbb{T}^{2}\right)$ given by

$$
e_{k l}^{1} \mapsto\left(\begin{array}{c}
1 \\
0 \\
0 \\
0
\end{array}\right) \otimes e_{k l}, e_{k l}^{2} \mapsto\left(\begin{array}{c}
0 \\
1 \\
0 \\
0
\end{array}\right) \otimes e_{k l}, e_{k l}^{3} \mapsto\left(\begin{array}{c}
0 \\
0 \\
1 \\
0
\end{array}\right) \otimes e_{k l}, e_{k l}^{4} \mapsto\left(\begin{array}{c}
0 \\
0 \\
0 \\
1
\end{array}\right) \otimes e_{k l},
$$

$U_{1}, U_{2}, V_{t}$ and the above commutators can be written as follows:

$$
U_{1}=\mathbf{1} \otimes s_{1}, U_{2}=\mathbf{1} \otimes s_{2}, V_{t}=\mathbf{1} \otimes v_{a b t},
$$

where $s_{1} e_{k l}=e_{k+1, l}, s_{2} e_{k l}=e_{k, l+1}, v_{a b t} e_{k l}=\mathrm{e}^{\mathrm{i}(a k+b l) t} e_{k l}$, and

$$
\left[\tilde{Q}, U_{1}\right]=\left(\begin{array}{rrrr}
0 & a & -b & 0 \\
-a & 0 & 0 & b \\
b & 0 & 0 & a \\
0 & -b & -a & 0
\end{array}\right) \otimes s_{1}, \quad\left[\tilde{Q}, U_{2}\right]=\left(\begin{array}{rrrr}
0 & b & a & 0 \\
-b & 0 & 0 & -a \\
-a & 0 & 0 & b \\
0 & a & -b & 0
\end{array}\right) \otimes s_{2} .
$$

Using this representation, together with $\left[s_{1}, s_{2}\right]=0, s_{1} v_{a b t}=e^{i a t} v_{a b t} s_{1}, s_{2} v_{a b t}=e^{i b t} v_{a b t} s_{2}$, it is easy to show

LEMMA 1.

$$
\begin{gathered}
U_{j}\left[\tilde{Q}, U_{k}\right]=\left[\tilde{Q}, U_{k}\right] U_{j}, \quad \forall j, k \in\{1,2\}, \\
V_{t}\left[\tilde{Q}, U_{1}\right]=e^{i a t}\left[\tilde{Q}, U_{1}\right] V_{t}, \quad V_{t}\left[\tilde{Q}, U_{2}\right]=e^{i b t}\left[\tilde{Q}, U_{2}\right] V_{t}
\end{gathered}
$$




$$
\begin{gathered}
{\left[\tilde{Q}, U_{1}\right]\left[\tilde{Q}, U_{2}\right]=-\left[\tilde{Q}, U_{2}\right]\left[\tilde{Q}, U_{1}\right]} \\
{\left[\tilde{Q}, V_{t}\right]=0 .}
\end{gathered}
$$

Explicitly, we have

$$
\left[\tilde{Q}, U_{1}\right]\left[\tilde{Q}, U_{2}\right]=\left(\begin{array}{rrrr}
0 & 0 & 0 & -1 \\
0 & 0 & -1 & 0 \\
0 & 1 & 0 & 0 \\
1 & 0 & 0 & 0
\end{array}\right) \otimes s_{1} s_{2} .
$$

Proposition 4. (i) $\Omega_{\tilde{Q}}^{1}\left(\mathcal{O}\left(\mathbb{T}^{2}\right) \rtimes \mathbb{R}\right)$ is a free left (and right) $\mathcal{O}\left(\mathbb{T}^{2}\right) \rtimes \mathbb{R}$-module with basis $\left\{d u_{1}, d u_{2}\right\}$. Its bimodule structure is determined by

$$
\begin{gathered}
u_{j} d u_{k}=d u_{k} u_{j}, \quad \forall j, k \in\{1,2\}, \\
v_{t} d u_{1}=e^{i a t} d u_{1} v_{t}, \quad v_{t} d u_{2}=e^{i b t} d u_{2} v_{t} .
\end{gathered}
$$

Moreover,

$$
d v_{t}=0 .
$$

(ii) $\Omega_{\tilde{Q}}^{2}\left(\mathcal{O}\left(\mathbb{T}^{2}\right) \rtimes \mathbb{R}\right)$ is a free left (and right) $\mathcal{O}\left(\mathbb{T}^{2}\right) \rtimes \mathbb{R}$-module with basis $\left\{d u_{1} d u_{2}\right\}$, with

$$
d u_{1} d u_{2}=-d u_{2} d u_{1} .
$$

(iii) $\Omega_{\tilde{Q}}^{k}\left(\mathcal{O}\left(\mathbb{T}^{2}\right) \rtimes \mathbb{R}\right)=0$ for $k \geq 3$.

Proof. Since the representation $\pi$ of $\mathcal{O}\left(\mathbb{T}^{2}\right) \rtimes \mathbb{R}$ in $L^{2}\left(\mathbb{T}^{2}, E\right)$ is faithful, the equations (3.17), (3.18) and (3.20) follow from (3.12)-(3.14), whereas (3.19) comes from (3.15). Now it is sufficient to show that every element of $\pi^{1}\left(\Omega^{1}\left(\mathcal{O}\left(\mathbb{T}^{2}\right) \rtimes \mathbb{R}\right)\right)$ is of the form $a_{1}\left[\tilde{Q}, U_{1}\right]+a_{2}\left[\tilde{Q}, U_{2}\right], \quad a_{1}, a_{2} \in \pi\left(\Omega^{1}\left(\mathcal{O}\left(\mathbb{T}^{2}\right) \rtimes \mathbb{R}\right)\right)$ and that from $a_{1}\left[\tilde{Q}, U_{1}\right]+a_{2}\left[\tilde{Q}, U_{2}\right]=0$ follows $a_{1}=a_{2}=0$. The first claim is immediate from the fact that $\pi\left(\mathcal{O}\left(\mathbb{T}^{2}\right) \rtimes \mathbb{R}\right)$ is generated by $U_{1}, U_{1}^{*}, U_{2}, U_{2}^{*}, V_{t}$ and from (3.15), (3.12)-(3.14). (Note that commutators $\left[\tilde{Q}, U_{j}^{*}\right]$ can be reduced to $\left[\tilde{Q}, U_{j}\right]$ using the Leibniz rule: From $0=\left[\tilde{Q}, U_{j}^{*} U_{j}\right]=$ $U_{j}^{*}\left[\tilde{Q}, U_{j}\right]+\left[\tilde{Q}, U_{j}^{*}\right] U_{j}$ follows $\left[\tilde{Q}, U_{j}^{*}\right]=-U_{j}^{*}\left[\tilde{Q}, U_{j}\right] U_{j}^{*}$. On the other hand, multiplying $\left[\tilde{Q}, U_{j}\right] U_{j}=U_{j}\left[\tilde{Q}, U_{j}\right]$ from both sides with $U_{j}^{*}$ gives $U_{j}^{*}\left[\tilde{Q}, U_{j}\right]=\left[\tilde{Q}, U_{j}\right] U_{j}^{*}$.) It remains to show linear independence. Assume

$$
\sum_{i j}\left(a_{i j} V_{t_{i j}} U_{1}^{i} U_{2}^{j}\left[\tilde{Q}, U_{1}\right]+b_{i j} V_{t_{i j}^{\prime}} U_{1}^{i} U_{2}^{j}\left[\tilde{Q}, U_{2}\right]\right)=0,
$$

$a_{i j}, b_{i j} \in \mathbb{C}$, finite summation over $i, j \in \mathbb{Z}$. Acting with this expression on the basis vector $e_{k l}^{1}$ gives

$$
\begin{aligned}
& a_{i j} e^{i t_{i j}((k+i+1) a+(l+j) b)}\left(a e_{k+i+1, l+j}^{2}-b e_{k+i+1, l+j}^{3}\right) \\
& =b_{i+1, j-1} e^{i t_{i+1, j-1}^{\prime}((k+i+1) a+(l+j) b)}\left(b e_{k+i+1, l+j}^{2}+a e_{k+i+1, l+j}^{3}\right)
\end{aligned}
$$

(now for fixed $i, j$ ), which means

$$
\begin{aligned}
& a a_{i j} e^{i t_{i j}((k+i+1) a+(l+j) b)}-b b_{i+1, j-1} e^{i t_{i+1, j-1}^{\prime}((k+i+1) a+(l+j) b)}=0, \\
& b a_{i j} e^{i t_{i j}((k+i+1) a+(l+j) b)}+a b_{i+1, j-1} e^{i t_{i+1, j-1}^{\prime}((k+i+1) a+(l+j) b)}=0 .
\end{aligned}
$$


Since

$$
\begin{gathered}
\operatorname{det}\left(\begin{array}{cc}
a e^{i t_{i j}((k+i+1) a+(l+j) b)} & -b e^{i t_{i+1, j-1}^{\prime}((k+i+1) a+(l+j) b)} \\
b e^{i t_{i j}((k+i+1) a+(l+j) b)} & a e^{i t_{i+1, j-1}^{\prime}((k+i+1) a+(l+j) b)}
\end{array}\right) \\
=\left(a^{2}+b^{2}\right) e^{i\left(t_{i j}+t_{i+1, j-1}^{\prime}\right)((k+i+1) a+(l+j) b)}=e^{i\left(t_{i j}+t_{i+1, j-1}^{\prime}\right)((k+i+1) a+(l+j) b)} \neq 0,
\end{gathered}
$$

this system has only the trivial solution $a_{i j}=b_{i+1, j-1}=0$. Thus (i) is proven.

To prove (ii) note that differentiating (3.17) for $j=k$ leads immediately to $d u_{1} d u_{1}=$ $d u_{2} d u_{2}=0$. Moreover, (3.14) yields $d u_{1} d u_{2}=-d u_{2} d u_{1}$, so that $\Omega_{\tilde{Q}}^{2}\left(\mathcal{O}\left(\mathbb{T}^{2}\right) \rtimes \mathbb{R}\right)$ is generated by $d u_{1} d u_{2}$. It remains to show that it is freely generated. To this end we have to determine $\operatorname{ker} \pi^{1}$. From (3.12) and (3.13) it follows that $\operatorname{ker} \pi^{1}$ contains the bimodule generated by the elements $u_{j} d u_{k}-d u_{k} u_{j}, v_{t} d u_{1}-e^{i a t} d u_{1} v_{t}, v_{t} d u_{2}-e^{i b t} d u_{2} v_{t}$. On the other hand, this bimodule also contains $\operatorname{ker} \pi^{1}$ : Let

$$
\alpha=\sum a_{t_{j} k l, t_{m} n q}^{r} v_{t_{j}} u_{1}^{k} u_{2}^{l} d u_{r} v_{t_{m}} u_{1}^{n} u_{2}^{q} \in \operatorname{ker} \pi^{1} .
$$

Using the commutation rules (3.12) and (3.13) and the basis property of the $\left[\tilde{Q}, u_{i}\right]$ already proved in (i), one concludes from $\pi^{1}(\alpha)=0$ that

$$
\sum a_{t_{j} k l, t_{m} n q}^{r} v_{t_{j}+t_{m}} u_{1}^{k+n} u_{2}^{l+q} e^{-i((k+1) a+l b) t_{m}}=0, \quad r=1,2 .
$$

Now, making use of the basis property of the monomials $v_{t} u_{1}^{k} u_{2}^{l}$ (Proposition 2), one has

$$
\sum_{t_{j}+t_{m}=T, k+n=K, l+q=L} a_{j k l, m n q}^{1} e^{-i((k+1) a+l b) t_{m}}=0 \quad(r=1),
$$

and a similar equation for $r=2$, for every fixed $T, K, L$. Now fix (for $r=1$ ) $k_{0}, l_{0}, t_{j_{0}}$ and write equation $(3.21)$ as

$$
\begin{aligned}
& a_{t_{j_{0}} k_{0} l_{0}, T-t_{j_{0}}, K-k_{0}, L-l_{0}}^{1}= \\
& \quad-e^{i\left(\left(k_{0}+1\right) a+l_{0} b\right)\left(T-t_{j_{0}}\right)} \sum_{t_{j} \neq t_{j_{0}}, k \neq k_{0}, l \neq l_{0}} a_{t_{j} k l, T-t_{j}, K-k, L-l}^{1} e^{-i((k+1) a+l b)\left(T-t_{j}\right)} .
\end{aligned}
$$

Inserting this and a similar expression for $r=2$ into $\alpha$ one obtains

$$
\begin{aligned}
& \alpha=\sum_{\left(t_{j}, k, l\right) \neq\left(t_{j_{0}}, k_{0}, l_{0}\right)} a_{t_{j} k l, T-t_{j}, K-k, L-l}^{1} v_{t_{j}} u_{1}^{k} u_{2}^{l} d u_{1} v_{T-t_{j}} u_{1}^{K-k} u_{2}^{L-l} \\
& -e^{i\left(\left(k_{0}+1\right) a+l_{0} b\right)\left(T-t_{j_{0}}\right)} \sum_{\left(t_{j}, k, l\right) \neq\left(t_{j_{0}}, k_{0}, l_{0}\right)} a_{t_{j} k l, T-t_{j}, K-k, L-l}^{1} v_{t_{j_{0}}} u_{1}^{k_{0}} u_{2}^{l_{0}} d u_{1} v_{T-t_{j_{0}}} u_{1}^{K-k_{0}} u_{2}^{L-l_{0}} \\
& \quad \cdot e^{i((k+1) a+l b)\left(T-t_{j}\right)}+(\text { a similar term for } r=2) .
\end{aligned}
$$

Now, fix $t_{j}>t_{j_{0}}, k>k_{0}, l>l_{0}$, and reduce the power of $u_{2}$ in front of $d u_{1}$ in the first term by subtracting and adding $d u_{1} u_{2}$, thus producing a term in the bimodule (with $u_{2} d u_{1}-d u_{1} u_{2}$ in the middle) and a new term with a new $e$-factor of the old kind. Iterating this procedure removes all superfluous powers of $u_{2}$. One can do the same for $u_{1}$ and $v_{t}$ and finally ends up with an expression which turns out to be zero (up to a lot of terms all lying in the bimodule). We leave the detailed computation to the reader. Thus we have shown that ker $\pi^{1}$ is also contained in the bimodule generated by the elements (3.17) and (3.18). 
Therefore, a general element of $\operatorname{ker} \pi^{1}$ is of the form

$$
j=\sum a_{k} \alpha_{k} b_{k}
$$

with $a_{k}, b_{k} \in \mathcal{O}\left(\mathbb{T}^{2}\right) \rtimes \mathbb{R}, \alpha_{k}$ one of the above generating elements of $\operatorname{ker} \pi^{1}$. Then

$$
\pi^{2}(d j)=\sum \pi\left(a_{k}\right) \pi^{2}\left(d \alpha_{k}\right) \pi\left(b_{k}\right),
$$

because the other terms appearing according to the Leibniz rule contain a factor $\pi^{1}\left(\alpha_{k}\right)$ $=0$. We have to determine $\pi^{2} \circ d\left(u_{j} d u_{k}-d u_{k} u_{j}\right)=\pi^{2}\left(d u_{j} d u_{k}+d u_{k} d u_{j}\right)=\left[\tilde{Q}, U_{j}\right]\left[\tilde{Q}, U_{k}\right]+$ $\left[\tilde{Q}, U_{k}\right]\left[\tilde{Q}, U_{j}\right]$. A trivial calculation using (3.11) and (3.10) shows that $\left[\tilde{Q}, U_{j}\right]\left[\tilde{Q}, U_{j}\right]=$ $-U_{j}^{2}$, whereas $\left[\tilde{Q}, U_{1}\right]\left[\tilde{Q}, U_{2}\right]+\left[\tilde{Q}, U_{2}\right]\left[\tilde{Q}, U_{1}\right]=0$ by $(3.14)$. It follows that

$$
\pi^{2}\left(d \operatorname{ker} \pi^{1}\right)=\pi\left(\mathcal{O}\left(\mathbb{T}^{2}\right) \rtimes \mathbb{R}\right)
$$

and it remains to show that from $\pi(a)\left[\tilde{Q}, U_{1}\right]\left[\tilde{Q}, U_{2}\right] \in \pi\left(\mathcal{O}\left(\mathbb{T}^{2}\right) \rtimes \mathbb{R}\right)$ follows $a=0$. Indeed, this follows from the fact that algebra elements have the diagonal form (3.10) whereas $\left[\tilde{Q}, U_{1}\right]\left[\tilde{Q}, U_{2}\right]$ is antidiagonal $(3.16)$, which is preserved under multiplication with a diagonal element $\pi(a)$.

(iii) is a trivial consequence of the fact that in a form of degree $\geq 3$ at least two differentials of the same generator $u_{j}$ will meet to produce 0 .

Note that one can define calculi $\Omega_{\tilde{Q}}^{1}(\mathcal{A})$ and $\Omega_{\tilde{Q}}^{2}(\mathcal{A})$ for the topological version $\mathcal{A}=$ $C^{\infty}\left(\mathbb{T}^{2}\right) \rtimes \mathbb{R}([18$, Remark 4$])$.

3.3. The mixed signature operator. Let us now consider the mixed signature operator $Q$ given by formula (2.5). In matrix representation, we have

$$
Q=\left(\begin{array}{cccc}
\frac{\partial^{2}}{\partial x^{2}} & 0 & \frac{\partial}{\partial y} & 0 \\
0 & -\frac{\partial^{2}}{\partial x^{2}} & 0 & -\frac{\partial}{\partial y} \\
-\frac{\partial}{\partial y} & 0 & -\frac{\partial^{2}}{\partial x^{2}} & 0 \\
0 & \frac{\partial}{\partial y} & 0 & \frac{\partial^{2}}{\partial x^{2}}
\end{array}\right)
$$

In order to diagonalize this operator, we have to solve the eigenvalue problem

$$
Q\left(\begin{array}{l}
f_{1} \\
f_{2} \\
f_{3} \\
f_{4}
\end{array}\right)=\lambda\left(\begin{array}{l}
f_{1} \\
f_{2} \\
f_{3} \\
f_{4}
\end{array}\right)
$$

with $f_{i} \in L^{2}\left(\mathbb{T}^{2}, E\right) . Q$ is already block-diagonal and acts in the same way in the space of $(0,0)$ - and $(0,1)$-forms and in the space of $(1,1)$ - and $(1,0)$-forms. It suffices to diagonalize one block. Defining

$$
g=f_{1}+f_{3}, \quad h=f_{1}-f_{3},
$$

one arrives at

$$
\frac{\partial^{2} h}{\partial x^{2}}+\frac{\partial h}{\partial y}=\lambda g, \quad \frac{\partial^{2} g}{\partial x^{2}}-\frac{\partial g}{\partial y}=\lambda h .
$$

Returning to the original coordinates $\left(\vartheta_{1}, \vartheta_{2}\right)$, the foregoing equations read

$$
a^{2} \frac{\partial^{2} h}{\partial \vartheta_{1}^{2}}+2 a b \frac{\partial^{2} h}{\partial \vartheta_{1} \partial \vartheta_{2}}+b^{2} \frac{\partial^{2} h}{\partial \vartheta_{2}^{2}}-b \frac{\partial h}{\partial \vartheta_{1}}+a \frac{\partial h}{\partial \vartheta_{2}}=\lambda g
$$




$$
a^{2} \frac{\partial^{2} g}{\partial \vartheta_{1}^{2}}+2 a b \frac{\partial^{2} g}{\partial \vartheta_{1} \partial \vartheta_{2}}+b^{2} \frac{\partial^{2} g}{\partial \vartheta_{2}^{2}}+b \frac{\partial g}{\partial \vartheta_{1}}-a \frac{\partial g}{\partial \vartheta_{2}}=\lambda h
$$

The ansatz

$$
g=\sum_{k, l \in \mathbb{Z}} \eta_{k l} e^{i\left(k \vartheta_{1}+l \vartheta_{2}\right)}, \quad h=\sum_{k, l \in \mathbb{Z}} \chi_{k l} e^{i\left(k \vartheta_{1}+l \vartheta_{2}\right)}
$$

leads to

$$
\left(\left(a^{2} k^{2}+2 a b k l+b^{2} l^{2}\right)^{2}+(b k-a l)^{2}\right) \chi_{k l}=\lambda^{2} \chi_{k l},
$$

which gives the eigenvalues

$$
\lambda_{k l \pm}= \pm \sqrt{(a k+b l)^{4}+(b k-a l)^{2}} .
$$

One easily concludes that eigenvectors to the eigenvalues $\lambda_{k l \pm}$ are of the form

$$
h_{k l}=e_{k l}, \quad g_{k l \pm}=\gamma_{k l \pm} e_{k l}
$$

with

$$
\gamma_{k l \pm}=\frac{-(a k+b l)^{2}+i(a l-b k)}{\lambda_{k l \pm}} .
$$

The eigenvectors of the original problem (3.22) are

$$
\begin{aligned}
& f_{1 k l \pm}=\frac{1}{2}\left(g_{k l \pm}+h_{k l}\right)=\frac{1}{2}\left(1+\gamma_{k l \pm}\right) e_{k l}, \\
& f_{3 k l \pm}=\frac{1}{2}\left(g_{k l \pm}-h_{k l}\right)=\frac{1}{2}\left(\gamma_{k l \pm}-1\right) e_{k l},
\end{aligned}
$$

or, written as elements of $L^{2}\left(\mathbb{T}^{2}, E\right)$,

$$
e_{k l \pm}^{(1)}=\frac{1}{2} e_{k l}\left(\left(\gamma_{k l \pm}+1\right) \mathbf{1}+\left(\gamma_{k l \pm}-1\right) \nu\right) .
$$

Since the metrics are chosen so that the frame elements $1, \tau, \nu, \tau \otimes \nu$ are orthonormal, these vectors are already orthonormal (note that $\left|\gamma_{k l \pm}\right|=1$.) The same argument yields another set

$$
e_{k l \pm}^{(2)}=\frac{1}{2} e_{k l}\left(\left(\gamma_{k l \pm}+1\right) \tau \otimes \nu+\left(\gamma_{k l \pm}-1\right) \tau\right)
$$

of eigenvectors to the same eigenvalues $\lambda_{k l \pm}$. Note that the eigenvalue 0 appears only for $k=l=0$. In that case, equations (3.23) and (3.24) decouple, and we get four independent eigenvectors $\mathbf{1}, \tau, \nu, \tau \otimes \nu$. In order to see that these vectors together with the $e_{k l \pm}^{(1,2)}$ form an orthonormal basis of $L^{2}\left(\mathbb{T}^{2}, E\right)$, it is sufficient to see that all the vectors $e_{k l} \mathbf{1}, e_{k l} \tau, e_{k l} \nu, \quad e_{k l} \tau \otimes \nu$ are linear combinations of the foregoing vectors. This follows from the fact that the matrix

$$
\left(\begin{array}{cc}
\gamma_{k l+}+1 & \gamma_{k l+}-1 \\
\gamma_{k l-}+1 & \gamma_{k l-}-1
\end{array}\right)
$$

is always invertible (its determinant being $-4 \gamma_{k l+}$ ).

Thus we have found the spectral decomposition of the selfadjoint operator $Q$. Its unboundedness is reflected in the unboundedness of the $\lambda_{k l \pm}$. It is now easy to write down also the spectral decomposition of the corresponding Dirac operator $D$ : Applying (2.7) for nonzero eigenvalues gives

$$
D e_{k l \pm}^{(1,2)}= \pm \sqrt{\lambda_{k l}} e_{k l \pm}^{(1,2)}
$$


where $\lambda_{k l}$ is the positive root $\lambda_{k l+}$. Putting $e_{00+}^{(1)}=\mathbf{1}, e_{00-}^{(1)}=\nu, e_{00+}^{(2)}=\tau \otimes \nu$ and $e_{00-}^{(2)}=\tau$, the formula defines $D$ also on the kernel of $Q$ (cf. 2.6), and gives the spectral decomposition of $D$.

The unitary operators $U_{1}, U_{2}$ and $V_{t}$ act by (3.2) on the basis vectors $e_{k l \pm}^{(1,2)}$ as follows:

$$
\begin{aligned}
U_{1} e_{k l \pm}^{(1,2)} & =\frac{1}{2}\left\{\left(1+\frac{\gamma_{k l \pm}}{\gamma_{k+1, l+}}\right) e_{k+1, l+}^{(1,2)}+\left(1+\frac{\gamma_{k l \pm}}{\gamma_{k+1, l-}}\right) e_{k+1, l-}^{(1,2)}\right\} \\
U_{2} e_{k l \pm}^{(1,2)} & =\frac{1}{2}\left\{\left(1+\frac{\gamma_{k l \pm}}{\gamma_{k, l+1,+}}\right) e_{k, l+1,+}^{(1,2)}+\left(1+\frac{\gamma_{k l \pm}}{\gamma_{k, l+1,-}}\right) e_{k, l+1,-}^{(1,2)}\right\} \\
V_{t} e_{k l \pm}^{(1,2)} & =\mathrm{e}^{\mathrm{i}(k a+l b) t} e_{k l \pm}^{(1,2)} .
\end{aligned}
$$

Defining

$$
\eta_{k l \pm}^{(1,2)}:=\frac{1}{2}\left(e_{k l+}^{(1,2)} \pm e_{k l-}^{(1,2)}\right)
$$

one finds

$$
\begin{aligned}
& U_{1} \eta_{k l+}^{(1,2)}=\eta_{k+1, l+}^{(1,2)}, \\
& U_{1} \eta_{k l-}^{(1,2)}=\frac{\gamma_{k l}}{\gamma_{k+1, l}} \eta_{k+1, l-}^{(1,2)}, \\
& U_{2} \eta_{k l+}^{(1,2)}=\eta_{k, l+1,+}^{(1,2)}, \\
& U_{2} \eta_{k l-}^{(1,2)}=\frac{\gamma_{k l}}{\gamma_{k, l+1}} \eta_{k, l+1,-}^{(1,2)}, \\
& V_{t} \eta_{k l \pm}^{(1,2)}=e^{i(k a+l b) t} \eta_{k l \pm}^{(1,2)}, \\
& D \eta_{k l \pm}^{(1,2)}=\sqrt{\lambda_{k l}} \eta_{k l \mp}^{(1,2)} .
\end{aligned}
$$

From Theorem 1 or by direct computation using (3.25)-(3.30) one gets

Proposition 5. $\left(C^{\infty}\left(\mathbb{T}^{2}\right) \rtimes \mathbb{R}, L^{2}\left(\mathbb{T}^{2}, E\right), D\right)$ is a spectral triple of dimension 3.

Next, one would like to describe the differential calculus $\Omega_{D}$ related to this spectral triple. Unfortunately, we have no definite result about $\Omega_{D}$. We will however show that the first order calculus for the restriction of the spectral triple to the subalgebra $C^{\infty}\left(\mathbb{T}^{2}\right)$ is the universal one, supporting our conjecture that also the first order calculus of the full triple is universal, up to some relations involving $V_{t}$. To begin with, we have as an immediate consequence of (3.25)-(3.30)

Lemma 2. Let $p, q, r, s \in \mathbb{Z}$. Then we have

$$
U_{1}^{r} U_{2}^{p}\left[D, U_{1}^{s} U_{2}^{q}\right] \eta_{k l \pm}^{(1,2)}=\frac{\sqrt{\lambda_{k+s, l+q}} \gamma_{k+s, l+q}-\sqrt{\lambda_{k l}} \gamma_{k l}}{\gamma_{k+r+s, l+p+q}} \eta_{k+r+s, l+p+q \mp}^{(1,2)} .
$$

Moreover,

$$
\left[D, V_{t}\right]=0, \quad V_{t}\left[D, U_{1}\right]=\mathrm{e}^{\mathrm{i} a t}\left[D, U_{1}\right] V_{t}, \quad V_{t}\left[D, U_{2}\right]=\mathrm{e}^{\mathrm{i} b t}\left[D, U_{2}\right] V_{t} .
$$

Proof. By direct computation using (3.25)-(3.30).

From Theorem 1 we know that the particular choice $\Gamma=\mathbf{1}$ gives rise to a spectral triple over $C^{\infty}\left(\mathbb{T}^{2}\right)$. Let us now first investigate the corresponding differential calculus 
$\Omega_{D}\left(\mathcal{O}\left(\mathbb{T}^{2}\right)\right)$. By faithfulness of the representation, we can again identify $\Omega_{D}^{1}\left(\mathcal{O}\left(\mathbb{T}^{2}\right)\right)$ with a subspace of $B\left(L^{2}(\mathcal{H})\right)$. We have

Proposition 6. The first order differential calculus $\Omega_{D}^{1}\left(\mathcal{O}\left(\mathbb{T}^{2}\right)\right)$ is freely generated by the elements $\left[D, U_{1}^{s} U_{2}^{q}\right](s, q \in \mathbb{Z})$.

Proof. We show that no nontrivial relations between $U_{1}^{s} U_{2}^{q}$ and commutators $\left[D, U_{1}^{t} U_{2}^{r}\right]$ exist. Let us first consider relations involving $D$ and $U_{1}$ only. From Lemma 2 it follows for $p=q=0$ that

$$
U_{1}^{r}\left[D, U_{1}^{s}\right] \eta_{k l \pm}^{(1,2)}=\frac{\sqrt{\lambda_{k+s, l}} \gamma_{k+s, l}-\sqrt{\lambda_{k l}} \gamma_{k l}}{\gamma_{k+r+s, l}} \eta_{k+r+s, l \mp}^{(1,2)} .
$$

Using the Leibniz rule and the fact that different overall powers of $U_{1}$ are independent (by the foregoing formula) we find that nontrivial relations would be of the form

$$
\sum_{m=0}^{s-1} a_{m} U_{1}^{m}\left[D, U_{1}^{s-m}\right]=0
$$

for $s \in \mathbb{N}$. Applying (3.33) to $\eta_{n 0 \pm}^{(1,2)}(n=k, \ldots, k+s-1)$ we get, after a redefinition of the summation index, the following system of equations:

$$
\begin{array}{r}
\sum_{j=0}^{s-1} a_{j}\left(\sqrt{\lambda_{k+j+1,0}} \gamma_{k+j+1,0}-\sqrt{\lambda_{k 0}} \gamma_{k 0}\right)=0, \\
\vdots \\
\sum_{j=0}^{s-1} a_{j}\left(\sqrt{\lambda_{k+j+s, 0}} \gamma_{k+j+s, 0}-\sqrt{\lambda_{k+s-1,0}} \gamma_{k+s-1,0}\right)=0 .
\end{array}
$$

For the discussion of this system of equations it is useful to define a function $h$ on $\mathbb{Z}$ putting

$$
h(i)=\sqrt{\lambda_{i 0}} \gamma_{i 0}-\sqrt{\lambda_{i-1,0}} \gamma_{i-1,0} .
$$

In terms of the function $h$, the matrix of the system of equations (3.34) is

$$
\left(\begin{array}{llll}
h(k+1) & h(k+2)+h(k+1) & \cdots & h(k+s)+\ldots+h(k+1) \\
h(k+2) & h(k+3)+h(k+2) & \cdots & h(k+s+1)+\ldots+h(k+2) \\
& \vdots & & \\
h(k+s) & h(k+s+1)+h(k+s) & \cdots & h(k+2 s-1)+\ldots+h(k+s)
\end{array}\right) .
$$

The determinant of this matrix can be tranformed into the following expression, using multilinearity and antisymmetry of the columns:

$$
\left|\begin{array}{llll}
h(k+1) & h(k+2) & \cdots & h(k+s) \\
h(k+2) & h(k+3) & \cdots & h(k+s+1) \\
& \vdots & & \\
h(k+s) & h(k+s+1) & \cdots & h(k+2 s-1)
\end{array}\right| .
$$

It is sufficient to show that the determinant (3.35) is always nonzero. Indeed: 
LEMMA 3. We have

$$
\left|\begin{array}{ccc}
h\left(i_{0}\right) & \cdots & h\left(i_{0}+k\right) \\
\vdots & \ddots & \vdots \\
h\left(i_{k}\right) & \cdots & h\left(i_{k}+k\right)
\end{array}\right| \neq 0
$$

for all $k \in \mathbb{N}$ and $i_{0}, \ldots, i_{k} \in \mathbb{Z}$.

Proof. See Appendix A.

Thus, there are no relations between $U_{1}$ and $D$ besides the ones coming from the Leibniz rule. In the general case we are looking for $a_{m n} \in \mathbb{C}$ such that

$$
\sum_{m=0}^{s-1} \sum_{n=0}^{q-1} a_{m n} U_{1}^{m} U_{2}^{n}\left[D, U_{1}^{s-m} U_{2}^{q-n}\right]=0 .
$$

Again, we are led to the consideration of a homogeneous linear system of equations for the $a_{m n}$. The corresponding matrix of coefficients is an $(s q) \times(s q)$-matrix with general matrix element

$$
C_{k,(m, n)}=\left(\sqrt{\lambda_{k+s-m, q-n}} \gamma_{k+s-m, q-n}-\sqrt{\lambda_{k 0}} \gamma_{k 0}\right),
$$

$(k=1, \ldots, s q$.$) In analogy to the case discussed above we have$

Lemma 4. Let $s, q \in \mathbb{N}$ be fixed. Then we have

$$
\operatorname{det}\left(C_{k,(m, n)}\right) \neq 0 \text {. }
$$

Proof. The proof is a straightforward generalization of the proof of Lemma 3 to the case of functions, defined on $\mathbb{Z}^{2}$, see [21].

The proof of the proposition follows now immediately from the fact that between the elements $\left[D, U_{1}^{s} U_{2}^{q}\right]$ there are no relations besides the ones coming from the Leibniz rule.

We were not able to derive more relations of the type (3.32) between commutators of $D$ with some generator and other generators (up to such relations resulting from applying $[D, \cdot]$ to $(3.3)-(3.6)$ and the unitarity condition, using the derivation property). This seems to be due to the fact that $\lambda_{k l}$ and $\gamma_{k l}$ contain second and fourth powers of $k$ and $l$ under the square root. Therefore we have

Conjecture 1. The bimodule $\Omega_{D}^{1}\left(C^{\infty}\left(\mathbb{T}^{2}\right) \rtimes \mathbb{R}\right)$ is generated by $d u_{1}$ and $d u_{2}$ and is described by two relations,

$$
v_{t} d u_{1}=e^{i a t} d u_{1} v_{t}, \quad v_{t} d u_{2}=e^{i b t} d u_{2} v_{t} .
$$

It seems that these difficulties in the end come from the quadratic part in the signature operator $Q$.

Let us note that we could choose another diffeomorphism group, restricting the action of $\mathbb{R}$ to the subgroup $\mathbb{Z}$. Then, the generators $V_{t}$ (or $v_{t}$ ) would be reduced to one generator $V_{1}=V\left(v_{1}=v\right)$, and all the above formulae remain, replacing always $V_{t}\left(v_{t}\right)$ by some power of $V(v)$. However, we would not get rid of the difficulties related to the differential calculus. 
Acknowledgements. The authors are grateful to K. Schmüdgen for helpful discussions. R. M. was supported by the Deutsche Forschungsgemeinschaft. Part of the work was done during his stay at the Max-Planck-Institute for Mathematics in the Sciences in Leipzig.

A. Proof of Lemma 3. The proof of this lemma rests on the following characterization of functions $f$ defined by determinants of Hankelian type, see [23], such that

$$
\left|\begin{array}{ccc}
f\left(i_{0}\right) & \cdots & f\left(i_{0}+k\right) \\
\vdots & \ddots & \vdots \\
f\left(i_{k}\right) & \cdots & f\left(i_{k}+k\right)
\end{array}\right|=0
$$

$\forall k \in \mathbb{N}$ and $i_{0}, \ldots, i_{k} \in \mathbb{Z}$. We have

THEOREM 2. A function $f$ defined on $\mathbb{Z}$ fulfils (A.1) if and only if it is of one of the following two types:

$$
\begin{aligned}
& f_{1}(i)=\beta^{i} \sum_{j=0}^{k-1} \alpha_{j} i^{j} \\
& f_{2}(i)=\sum_{j=1}^{k} \alpha_{j} \beta_{j}^{i}
\end{aligned}
$$

with $\alpha, \beta, \beta_{j} \in \mathbb{C}$.

Proof. Let us first show by induction that $f_{1}$ and $f_{2}$ fulfil (A.1). For $f_{1}(i)=$ $\beta^{i} \sum_{j=0}^{k-1} \alpha_{j} i^{j}$ and $k=1$ we have

$$
\left|\begin{array}{cc}
\alpha_{0} \beta^{i} & \alpha_{0} \beta^{i+1} \\
\alpha_{0} \beta^{j} & \alpha_{0} \beta^{j+1}
\end{array}\right|=0,
$$

$\forall i, j \in \mathbb{Z}$. Let now (A.1) be valid for $k=n$. Then we have for $k=n+1$

$$
\begin{gathered}
\left|\begin{array}{cccc}
\beta^{i_{0}} \sum_{j=0}^{n} \alpha_{j} i_{0}^{j} & \beta^{i_{0}+1} \sum_{j=0}^{n} \alpha_{j}\left(i_{0}+1\right)^{j} & \cdots & \beta^{i_{0}+n+1} \sum_{j=0}^{n} \alpha_{j}\left(i_{0}+n+1\right)^{j} \\
\beta^{i_{1}} \sum_{j=0}^{n} \alpha_{j} i_{1}^{j} & \beta^{i_{1}+1} \sum_{j=0}^{n} \alpha_{j}\left(i_{1}+1\right)^{j} & \cdots & \beta^{i_{1}+n+1} \sum_{j=0}^{n} \alpha_{j}\left(i_{1}+n+1\right)^{j} \\
\vdots & \ddots & \ddots & \vdots \\
\beta^{i_{n+1}} \sum_{j=0}^{n} \alpha_{j} i_{n+1}^{j} & \beta^{i_{n+1}+1} \sum_{j=0}^{n} \alpha_{j}\left(i_{n+1}+1\right)^{j} & \cdots & \beta^{i_{n+1}+n+1} \sum_{j=0}^{n} \alpha_{j}\left(i_{n+1}+n+1\right)^{j}
\end{array}\right|= \\
\beta^{i_{0}+\cdots+i_{n+1}+\frac{(n+1)(n+2)}{2}}\left|\begin{array}{cccc}
\sum_{j=0}^{n} \alpha_{j} i_{0}^{j} & \sum_{j=0}^{n} \alpha_{j}\left(i_{0}+1\right)^{j} & \cdots & \sum_{j=0}^{n} \alpha_{j}\left(i_{0}+n+1\right)^{j} \\
\sum_{j=0}^{n} \alpha_{j} i_{1}^{j} & \sum_{j=0}^{n} \alpha_{j}\left(i_{1}+1\right)^{j} & \cdots & \sum_{j=0}^{n} \alpha_{j}\left(i_{1}+n+1\right)^{j} \\
\vdots & \vdots & \ddots & \vdots \\
\sum_{j=0}^{n} \alpha_{j} i_{n+1}^{j} & \sum_{j=0}^{n} \alpha_{j}\left(i_{n+1}+1\right)^{j} & \cdots & \sum_{j=0}^{n} \alpha_{j}\left(i_{n+1}+n+1\right)^{j}
\end{array}\right|
\end{gathered}
$$


But

$$
\begin{aligned}
& \left|\begin{array}{cccc}
\sum_{j=0}^{n} \alpha_{j} i_{0}^{j} & \sum_{j=0}^{n} \alpha_{j}\left(i_{0}+1\right)^{j} & \ldots & \sum_{j=0}^{n} \alpha_{j}\left(i_{0}+n+1\right)^{j} \\
\sum_{j=0}^{n} \alpha_{j} i_{1}^{j} & \sum_{j=0}^{n} \alpha_{j}\left(i_{1}+1\right)^{j} & \ldots & \sum_{j=0}^{n} \alpha_{j}\left(i_{1}+n+1\right)^{j} \\
\vdots & \vdots & \ddots & \vdots \\
\sum_{j=0}^{n} \alpha_{j} i_{n+1}^{j} & \sum_{j=0}^{n} \alpha_{j}\left(i_{n+1}+1\right)^{j} & \ldots & \sum_{j=0}^{n} \alpha_{j}\left(i_{n+1}+n+1\right)^{j}
\end{array}\right|= \\
& \sum_{j=0}^{n} \alpha_{j} i_{0}^{j} \quad \sum_{j=1}^{n} \alpha_{j}\left[\left(i_{0}+1\right)^{j}-i_{0}^{j}\right] \quad \cdots \quad \sum_{j=1}^{n} \alpha_{j}\left[\left(i_{0}+n+1\right)^{j}-\left(i_{0}+n\right)^{j}\right] \\
& \sum_{j=0}^{n} \alpha_{j} i_{1}^{j} \quad \sum_{j=1}^{n} \alpha_{j}\left[\left(i_{1}+1\right)^{j}-i_{1}^{j}\right] \quad \ldots \quad \sum_{j=1}^{n} \alpha_{j}\left[\left(i_{1}+n+1\right)^{j}-\left(i_{1}+n\right)^{j}\right]
\end{aligned}
$$

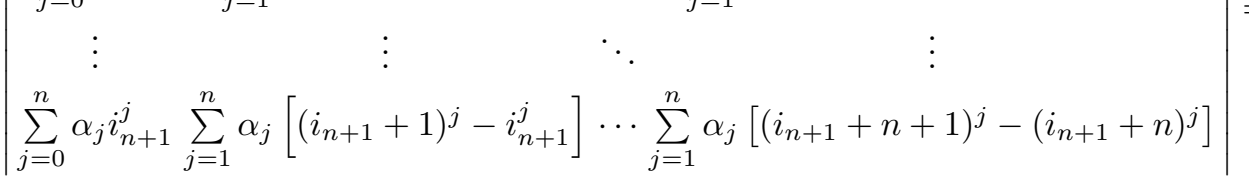

$$
\begin{aligned}
& \sum_{j=0}^{n} \alpha_{j} i_{0}^{j} \quad \sum_{j=1}^{n} \alpha_{j} \sum_{l=0}^{j-1}\left(\begin{array}{l}
j \\
l
\end{array}\right) i_{0}^{l} \quad \cdots \quad \sum_{j=1}^{n} \alpha_{j} \sum_{l=0}^{j-1}\left(\begin{array}{l}
j \\
l
\end{array}\right)\left(i_{0}+n\right)^{l} \\
& \sum_{j=0}^{n} \alpha_{j} i_{1}^{j} \quad \sum_{j=1}^{n} \alpha_{j} \sum_{l=0}^{j-1}\left(\begin{array}{l}
j \\
l
\end{array}\right) i_{1}^{l} \quad \cdots \quad \sum_{j=1}^{n} \alpha_{j} \sum_{l=0}^{j-1}\left(\begin{array}{l}
j \\
l
\end{array}\right)\left(i_{1}+n\right)^{l}= \\
& \left|\sum_{j=0}^{n} \alpha_{j} i_{n+1}^{j} \sum_{j=1}^{n} \alpha_{j} \sum_{l=0}^{j-1}\left(\begin{array}{l}
j \\
l
\end{array}\right) i_{n+1}^{l} \cdots \sum_{j=1}^{n} \alpha_{j} \sum_{l=0}^{j-1}\left(\begin{array}{l}
j \\
l
\end{array}\right)\left(i_{n+1}+n\right)^{l}\right| \\
& \sum_{j=0}^{n} \alpha_{j} i_{0}^{j}\left|\begin{array}{ccc}
\sum_{j=1}^{n} \alpha_{j} \sum_{l=0}^{j-1}\left(\begin{array}{l}
j \\
l
\end{array}\right) i_{1}^{l} & \cdots & \sum_{j=1}^{n} \alpha_{j} \sum_{l=0}^{j-1}\left(\begin{array}{l}
j \\
l
\end{array}\right)\left(i_{1}+n\right)^{l} \\
\vdots & \ddots & \vdots \\
\sum_{j=1}^{n} \alpha_{j} \sum_{l=0}^{j-1}\left(\begin{array}{l}
j \\
l
\end{array}\right) i_{n+1}^{l} & \cdots & \sum_{j=1}^{n} \alpha_{j} \sum_{l=0}^{j-1}\left(\begin{array}{l}
j \\
l
\end{array}\right)\left(i_{n+1}+n\right)^{l}
\end{array}\right|+\cdots+ \\
& (-1)^{n+1} \sum_{j=0}^{n} \alpha_{j} i_{n+1}^{j}\left|\begin{array}{ccc}
\sum_{j=1}^{n} \alpha_{j} \sum_{l=0}^{j-1}\left(\begin{array}{l}
j \\
l
\end{array}\right) i_{0}^{l} & \cdots & \sum_{j=1}^{n} \alpha_{j} \sum_{l=0}^{j-1}\left(\begin{array}{l}
j \\
l
\end{array}\right)\left(i_{0}+n\right)^{l} \\
\vdots & \ddots & \vdots \\
\sum_{j=1}^{n} \alpha_{j} \sum_{l=0}^{j-1}\left(\begin{array}{l}
j \\
l
\end{array}\right) i_{n}^{l} \cdots & \sum_{j=1}^{n} \alpha_{j} \sum_{l=0}^{j-1}\left(\begin{array}{l}
j \\
l
\end{array}\right)\left(i_{n}+n\right)^{l}
\end{array}\right|=0,
\end{aligned}
$$

by assumption. Analogously, for $f_{2}(i)=\sum_{j=1}^{k} \alpha_{j} \beta_{j}^{i}$ we have for $k=1$

$$
\left|\begin{array}{cc}
\alpha_{1} \beta_{1}^{i} & \alpha_{1} \beta_{1}^{i+1} \\
\alpha_{1} \beta_{1}^{j} & \alpha_{1} \beta_{1}^{j+1}
\end{array}\right|=0,
$$

$\forall i, j \in \mathbb{Z}$. Let us now assume the validity of (A.1) for $k=n$. Then we have for $k=n+1$ 


$$
\begin{aligned}
& \left|\begin{array}{cccc}
\sum_{j=1}^{n+1} \alpha_{j} \beta_{j}^{i_{0}} & \sum_{j=1}^{n+1} \alpha_{j} \beta_{j}^{i_{0}+1} & \ldots & \sum_{j=1}^{n+1} \alpha_{j} \beta_{j}^{i_{0}+n+1} \\
\sum_{j=1}^{n+1} \alpha_{j} \beta_{j}^{i_{1}} & \sum_{j=1}^{n+1} \alpha_{j} \beta_{j}^{i_{1}+1} & \ldots & \sum_{j=1}^{n+1} \alpha_{j} \beta_{j}^{i_{1}+n+1} \\
\vdots & \vdots & \ddots & \vdots \\
\sum_{j=1}^{n+1} \alpha_{j} \beta_{j}^{i_{n+1}} & \sum_{j=1}^{n+1} \alpha_{j} \beta_{j}^{i_{n+1}+1} & \cdots & \sum_{j=1}^{n+1} \alpha_{j} \beta_{j}^{i_{n+1}+n+1}
\end{array}\right|= \\
& \left|\begin{array}{cccc}
\sum_{j=1}^{n+1} \alpha_{j} \beta_{j}^{i_{0}} & \sum_{j=1}^{n} \alpha_{j} \beta_{j}^{i_{0}}\left(\beta_{j}-\beta_{n+1}\right) & \cdots & \sum_{j=1}^{n} \alpha_{j} \beta_{j}^{i_{0}+n}\left(\beta_{j}-\beta_{n+1}\right) \\
\sum_{j=1}^{n+1} \alpha_{j} \beta_{j}^{i_{1}} & \sum_{j=1}^{n} \alpha_{j} \beta_{j}^{i_{1}}\left(\beta_{j}-\beta_{n+1}\right) & \cdots & \sum_{j=1}^{n} \alpha_{j} \beta_{j}^{i_{1}+n}\left(\beta_{j}-\beta_{n+1}\right) \\
\vdots & \vdots & \ddots & \vdots \\
\sum_{j=1}^{n+1} \alpha_{j} \beta_{j}^{i_{n+1}} & \sum_{j=1}^{n} \alpha_{j} \beta_{j}^{i_{n+1}}\left(\beta_{j}-\beta_{n+1}\right) & \cdots & \sum_{j=1}^{n} \alpha_{j} \beta_{j}^{i_{n+1}+n}\left(\beta_{j}-\beta_{n+1}\right)
\end{array}\right|= \\
& \sum_{j=1}^{n+1} \alpha_{j} \beta_{j}^{i_{0}}\left|\begin{array}{ccc}
\sum_{j=1}^{n} \alpha_{j} \beta_{j}^{i_{1}}\left(\beta_{j}-\beta_{n+1}\right) & \cdots & \sum_{j=1}^{n} \alpha_{j} \beta_{j}^{i_{1}+n}\left(\beta_{j}-\beta_{n+1}\right) \\
\vdots & \ddots & \vdots \\
\sum_{j=1}^{n} \alpha_{j} \beta_{j}^{i_{n+1}}\left(\beta_{j}-\beta_{n+1}\right) & \cdots & \sum_{j=1}^{n} \alpha_{j} \beta_{j}^{i_{n+1}+n}\left(\beta_{j}-\beta_{n+1}\right)
\end{array}\right|+\cdots+ \\
& (-1)^{n+1} \sum_{j=1}^{n+1} \alpha_{j} \beta_{j}^{i_{n+1}}\left|\begin{array}{ccc}
\sum_{j=1}^{n} \alpha_{j} \beta_{j}^{i_{0}}\left(\beta_{j}-\beta_{n+1}\right) & \cdots & \sum_{j=1}^{n} \alpha_{j} \beta_{j}^{i_{0}+n}\left(\beta_{j}-\beta_{n+1}\right) \\
\vdots & \ddots & \vdots \\
\sum_{j=1}^{n} \alpha_{j} \beta_{j}^{i_{n}}\left(\beta_{j}-\beta_{n+1}\right) & \cdots & \sum_{j=1}^{n} \alpha_{j} \beta_{j}^{i_{n}+n}\left(\beta_{j}-\beta_{n+1}\right)
\end{array}\right|=0,
\end{aligned}
$$

by assumption.

Let us now assume that a function $f$ defined on $\mathbb{Z}$ fulfils (A.1) for some $k \in \mathbb{N}$. We choose $i_{1}=i_{0}+1, \ldots, i_{k}=i_{0}+k$ and let $f\left(i_{0}\right), \ldots, f\left(i_{0}+2 k-1\right)$ denote the corresponding values of $f$. Then $f\left(i_{0}+2 k\right)$ has to fulfil

$$
\left|\begin{array}{cccc}
f\left(i_{0}\right) & f\left(i_{0}+1\right) & \cdots & f\left(i_{0}+k\right) \\
f\left(i_{0}+1\right) & f\left(i_{0}+2\right) & \cdots & f\left(i_{0}+k+1\right) \\
\vdots & \vdots & \ddots & \vdots \\
f\left(i_{0}+k\right) & f\left(i_{0}+1\right) & \cdots & f\left(i_{0}+2 k\right)
\end{array}\right|=0
$$

provided that

$$
\left|\begin{array}{cccc}
f\left(i_{0}\right) & f\left(i_{0}+1\right) & \cdots & f\left(i_{0}+k-1\right) \\
f\left(i_{0}+1\right) & f\left(i_{0}+2\right) & \cdots & f\left(i_{0}+k\right) \\
\vdots & \vdots & \ddots & \vdots \\
f\left(i_{0}+k-1\right) & f\left(i_{0}+k\right) & \cdots & f\left(i_{0}+2 k-2\right)
\end{array}\right| \neq 0 .
$$

(We may assume without loss of generality that (A.5) holds. In [21] it is shown that in the other case one is led to the case $k-1$.) From (A.4) and (A.5) we find that the $2 k$ 
values $f\left(i_{0}\right), \ldots, f\left(i_{0}+2 k-1\right)$ determine $f$ completely. Now we show that this function is either of type (A.3) or (A.2).

Let first the constants $f\left(i_{0}\right), \ldots, f\left(i_{0}+2 k-1\right)$ be such that the following condition holds:

$$
\sum_{j=0}^{l+1} \beta^{l+1-j} f(i+j)(-1)^{j}\left(\begin{array}{c}
l+1 \\
j
\end{array}\right)=0
$$

for some $\beta \in \mathbb{C}, l \in\{0, \ldots, k-1\}$ and all $i=i_{0}, \ldots, i_{0}+2 k-l-1$. We show that the corresponding function on $\mathbb{Z}$ is of the form (A.2). Suppose that $\beta \in \mathbb{C}$ is a solution of (A.6). Then we find constants $\alpha_{i}$ as follows. Defining $g(i):=\frac{f(i)}{\beta^{i}}(\beta \neq 0)$, we can always find $\alpha_{i}(i=0, \ldots, l)$ as solutions of the following linear system of equations:

$$
\begin{aligned}
g\left(i_{0}\right) & =\alpha_{0}+\alpha_{1} i_{0}+\cdots+\alpha_{l} i_{0}^{l}, \\
g\left(i_{0}+1\right) & =\alpha_{0}+\alpha_{1}\left(i_{0}+1\right)+\cdots+\alpha_{l}\left(i_{0}+1\right)^{l}, \\
& \vdots \\
g\left(i_{0}+l\right) & =\alpha_{0}+\alpha_{1}\left(i_{0}+l\right)+\cdots+\alpha_{l}\left(i_{0}+l\right)^{l}
\end{aligned}
$$

by

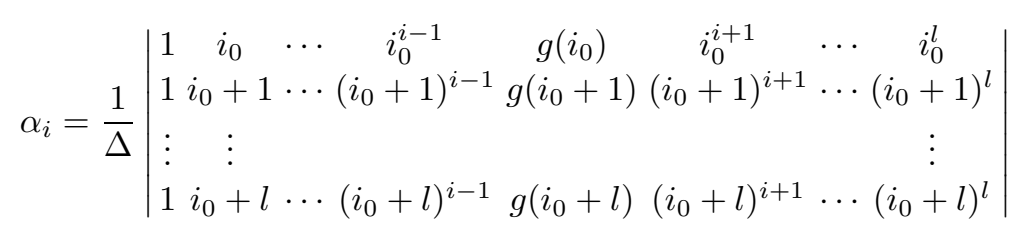

with

$$
\Delta=\left|\begin{array}{cccc}
1 & i_{0} & \cdots & i_{0}^{l} \\
1 & i_{0}+1 & \cdots & \left(i_{0}+1\right)^{l} \\
\vdots & \vdots & \vdots \\
1 & i_{0}+l & \cdots & \left(i_{0}+l\right)^{l}
\end{array}\right|=(-1)^{\frac{l(l+1)}{2}} \prod_{j=1}^{l} j ! \neq 0
$$

Now that we have chosen the constants $\beta$ and $\alpha_{0}, \ldots, \alpha_{l}$ such that

$$
f\left(i_{0}+j\right)=f_{1}\left(i_{0}+j\right)
$$

is fulfilled, for all $j=0, \ldots, l$, it remains to be shown that we also have

$$
f\left(i_{0}+l+1\right)=f_{1}\left(i_{0}+l+1\right) .
$$

But

$$
\sum_{j=0}^{r}(-1)^{j}\left(\begin{array}{l}
r \\
j
\end{array}\right) j^{s}=0
$$

$\forall s=0, \ldots, r-1$ (which follows from evaluating the $s$-th derivative of $f(x)=(x-1)^{r}=$ $\sum_{j=0}^{r}\left(\begin{array}{c}r \\ j\end{array}\right)(-1)^{j} x^{r-j}$ at $\left.x=1\right)$. Now we find

$$
\sum_{j=0}^{l+1} \beta^{l+1-j}(-1)^{j}\left(\begin{array}{c}
l+1 \\
j
\end{array}\right) f_{1}(i+j)=\sum_{j=0}^{l+1} \beta^{l+1-j} \beta^{i+j}(-1)^{j}\left(\begin{array}{c}
l+1 \\
j
\end{array}\right) \sum_{m=0}^{l} \alpha_{m}(i+j)^{m}
$$




$$
\begin{aligned}
& =\beta^{l+i+1} \sum_{j=0}^{l+1} \sum_{m=0}^{l} \sum_{n=0}^{m} \alpha_{m}(-1)^{j}\left(\begin{array}{c}
l+1 \\
j
\end{array}\right)\left(\begin{array}{c}
m \\
n
\end{array}\right) i^{n} j^{m-n} \\
& =\beta^{l+i+1} \sum_{m=0}^{l} \alpha_{m} \sum_{n=0}^{m}\left(\begin{array}{c}
m \\
n
\end{array}\right) i^{n} \sum_{j=0}^{l+1}(-1)^{j}\left(\begin{array}{c}
l+1 \\
j
\end{array}\right) j^{m-n}=0 .
\end{aligned}
$$

Therefore, we have

$$
\begin{aligned}
\sum_{j=0}^{l+1}(-1)^{j} \beta^{l+1-j}\left(\begin{array}{c}
l+1 \\
j
\end{array}\right)\left(f\left(i_{0}+j\right)\right. & \left.-f_{1}\left(i_{0}+j\right)\right) \\
& =(-1)^{l+1}\left(f\left(i_{0}+l+1\right)-f_{1}\left(i_{0}+l+1\right)\right)=0
\end{aligned}
$$

i.e.

$$
f\left(i_{0}+l+1\right)=f_{1}\left(i_{0}+l+1\right) .
$$

Let us now consider the general case (A.3). Suppose, that $f\left(i_{0}\right), \ldots, f\left(i_{0}+2 k-1\right)$ are chosen such that (A.6) does not hold. Then we have to solve the following system of algebraic equations (where we have chosen $i_{0}=0$ ):

$$
\begin{aligned}
f(0) & =C_{1}+\cdots+C_{k}, \\
f(1) & =C_{1} \beta_{1}+\cdots+C_{k} \beta_{k}, \\
\vdots & \\
f(2 k-1) & =C_{1} \beta_{1}^{2 k-1}+\cdots+C_{k} \beta_{k}^{2 k-1} .
\end{aligned}
$$

which can always be done using Gröbner basis techniques, see [21].

The proof of Lemma 3 follows now immediately from the observation that the function

$$
h(i)=\sqrt{\lambda_{i 0}} \gamma_{i 0}-\sqrt{\lambda_{i-1,0}} \gamma_{i-1,0}
$$

is obviously not of the form (A.2) or (A.3).

\section{References}

[1] N. Berline, E. Getzler and M. Vergne, Heat Kernels and Dirac Operators, Springer, Berlin, 1992.

[2] R. Bott, Lectures on characteristic classes and foliations, in: Lect. Notes Math. 279, Springer, Berlin, 1972, 1-94.

[3] A. H. Chamseddine and A. Connes, The spectral action principle, Comm. Math. Phys. 186 (1997), 731-750.

[4] A. Connes, Noncommutative Geometry, Academic Press, San Diego, 1994.

[5] A. Connes and J. Lott, Particle models and non-commutative geometry, Recent advances in field theory (Annecy-le-Vieux, 1990), Nucl. Phys. B Proc. Suppl. 18 B (1990), 29-47 (1991).

[6] A. Connes and H. Moscovici, The local index formula in noncommutative geometry, GAFA 5 (1995), 174-243.

[7] R. Coquereaux, G. Esposito-Farese and F. Scheck, Noncommutative geometry and graded algebras in electroweak interactions, Int. J. Mod. Phys. A, 7 (1992), 6555-6593. 
[8] J. Fröhlich, O. Grandjean and A. Recknagel, Supersymmetric quantum theory and differential geometry, Commun. Math. Phys. 193 (1998), 527-594.

[9] J. Fröhlich, O. Grandjean and A. Recknagel, Supersymmetric quantum theory and noncommutative geometry, Commun. Math. Phys. 203 (1999), 119-184.

[10] P. B. Gilkey, Invariance Theory, the Heat Equation, and the Atiyah-Singer Index Theorem, Publish or Perish, Wilmington, 1984.

[11] J. M. Gracia-Bondía, J .C. Várilly and H. Figueroa, Elements of Noncommutative Geometry, Birkhäuser, Basel, 2000.

[12] W. Kalau, N. A. Papadopoulos, J. Plass and J.-M. Warzecha, Differential algebras in non-commutative geometry, J. Geom. Phys. 16 (1995), 149-167.

[13] W. Kalau and M. Walze, Gravity, non-commutative geometry and the Wodzicki residue, J. Geom. Phys. 16 (1995), 327-344.

[14] D. Kastler, The Dirac operator and gravitation, Comm. Math. Phys. 166 (1995), 633-643.

[15] T. Krajewski, Classification of finite spectral triples, J. Geom. Phys. 28 (1998), 1-30.

[16] R. Matthes, G. Rudolph and R. Wulkenhaar, On the structure of a differential algebra used by Connes and Lott, Rep. Math. Phys. 38 (1996), 45-66.

[17] R. Matthes, G. Rudolph and R. Wulkenhaar, On a certain construction of graded Lie algebras with derivation, J. Geom. and Phys. 20 (1996), 107-141.

[18] R. Matthes, O. Richter and G. Rudolph, Spectral triples and differential calculi related to the Kronecker foliation, J. Geom. and Phys. 46 (2003), 48-73.

[19] P. Molino, Riemannian Foliations, Birkhäuser, Basel, 1988.

[20] M. Paschke and A. Sitarz, Discrete spectral triples and their symmetries, J. Math. Phys. 39 (1998), 6191-6205.

[21] O. Richter, On functions defined by the vanishing of determinants of Hankelian type, in preparation.

[22] M. A. Rieffel, $C^{*}$-algebras associated with irrational rotations, Pac. J. Math. 93 (1981), 415-429.

[23] R. Vein and P. Dale, Determinants and Their Applications in Mathematical Physics, Springer, Berlin, 1998. 